\title{
Enhancing semantic belief function to handle decision conflicts in SoS using k-means clustering
}

\author{
Eman K. Elsayed ${ }^{\text {Equal first author, 1,2 }}$, Ahmed Sharaf Eldin Ahmed ${ }^{3,4}$, Hebatullah Rashed Younes ${ }^{\text {Corresp. Equal first author, } 3}$ \\ 1 Mathematical and Computer Science Department, Faculty of Science, Al-Azhar University, (Girls Branch), Cairo, Egypt \\ 2 Computer Science Department, Faculty of Information Technology, Misr University for Science and Technology MUST, 6th of October, Egypt \\ 3 Information Technology Department, Faculty of Information Technology and Computer Science, Sinai University, Arish, Egypt \\ 4 Information System Department, Faculty of Computers and Artificial Intelligence, Helwan University, Cairo, Egypt \\ Corresponding Author: Hebatullah Rashed Younes \\ Email address: heba.rashed@su.edu.eg
}

Background: The endeavouring to offer complex special functions from individual systems gave rise to what is known as the System of Systems (SoS). SoS co-integrating systems together while allowing for absorbing more systems in the future. SoS as an integrated system simplifies operations, reduces costs, and ensures efficiency. However, conflict may result while co-integrating systems, violating the main benefits of SoS. This paper is concerned with enhancing the time required to detect and solve such conflicts.

Methods: We adopted the k-means clustering technique to enhance the detection and solving of conflict resulting while co-integrating new systems into an existing SoS. Instead of dealing with SoS as a single entity, we partition it into clusters. Each cluster contains nearby systems according to pre-specified criteria. We can consider each cluster a Sub SoS (S-SoS). By doing so, the conflict that may arise while co-integrating new systems can be detected and solved in a shorter time. We propose the Smart Semantic Belief Function Clustered System of Systems (SSBFCSoS) which is an enhancement of the Ontology Belief Function System of Systems (OBFSoS).

Results: The proposed method proved the ability to rapidly detect and resolve conflicts. It showed the ability to accommodate more systems as well, therefore, achieving the objectives of SoS. In order to test the applicability of the SSBFCSOS and compare its performance with other approaches, two datasets were employed. They are (Glest \& StarCraft Brood War). With each dataset, 15 test cases were examined. We achieved, on average, $89 \%$ in solving the conflict compared to $77 \%$ for other approaches. Moreover, it showed an acceleration of up to proportionality over previous approaches for about $16 \%$ in solving conflicts as well. Besides, it reduced the frequency of the same conflicts by approximately $23 \%$ better than the other method, not only in the same cluster but even while combining different clusters. 
1 Enhancing Semantic Belief Function to Handle

2 Decision Conflicts in SoS Using K-means Clustering

3

4

5

6

7

8

\section{Eman K. Elsayed ${ }^{1,2}$, Ahmed Sharaf Eldin Ahmed ${ }^{3,4}$, Hebatullah Rashed Younes ${ }^{3}$}

${ }^{1}$ Mathematical and Computer Science Department, Faculty of Science, Al-Azhar University, (Girls Branch), Cairo, Egypt

${ }^{2}$ Computer Science Department, Faculty of Information Technology, Misr University for Science and Technology MUST, $6^{\text {th }}$ of October, Egypt

${ }^{3}$ Information Technology Department, Faculty of Information Technology and Computer Science, Sinai University, Arish, Egypt

${ }^{4}$ Information System Department, Faculty of Computers and Artificial Intelligence, Helwan University, Cairo, Egypt

Corresponding Author:

Hebatullah Rashed Younes ${ }^{3}$

Mansoura, Egypt

Email address: heba.rashed@su.edu.eg

\section{Abstract}

Background: The endeavouring to offer complex special functions from individual systems gave rise to what is known as the System of Systems ( $\mathrm{SoS}$ ). SoS co-integrating systems together while allowing for absorbing more systems in the future. SoS as an integrated system simplifies operations, reduces costs, and ensures efficiency. However, conflict may result while cointegrating systems, violating the main benefits of SoS. This paper is concerned with enhancing the time required to detect and solve such conflicts.

Methods: We adopted the k-means clustering technique to enhance the detection and solving of conflict resulting while co-integrating new systems into an existing SoS. Instead of dealing with SoS as a single entity, we partition it into clusters. Each cluster contains nearby systems according to pre-specified criteria. We can consider each cluster a Sub SoS (S-SoS). By doing so, the conflict that may arise while co-integrating new systems can be detected and solved in a shorter time. We propose the Smart Semantic Belief Function Clustered System of Systems (SSBFCSoS) which is an enhancement of the Ontology Belief Function System of Systems (OBFSoS).

Results: The proposed method proved the ability to rapidly detect and resolve conflicts. It showed the ability to accommodate more systems as well, therefore, achieving the objectives of SoS. In order to test the applicability of the SSBFCSoS and compare its performance with other approaches, two datasets were employed. They are (Glest \& StarCraft Brood War). With each dataset, 15 test cases were examined. We achieved, on average, $89 \%$ in solving the conflict compared to $77 \%$ for other approaches. Moreover, it showed an acceleration of up to proportionality over previous approaches for about $16 \%$ in solving conflicts as well. Besides, it 
41 reduced the frequency of the same conflicts by approximately $23 \%$ better than the other method,

42 not only in the same cluster but even while combining different clusters.

43 Keywords System of Systems (SoS); Component Systems (CS); Conflict; Clustering; k-means.

44

45 Introduction

46 Recently and according to systems, it has been proven that in order to create a powerful, cointegrated, and multitasking system, an individual system will not be sufficient (Boehm and Lane 2007) (Robinson, Pawlowski et al. 2003) (Viana, Zisman et al. 2017). So, to achieve such a system, some systems must be co-integrated together in System of Systems (SoS) (Spanoudakis and Zisman 2001). SoS is a set of dedicated systems that have fetched their abilities to create a new complicated system, providing more performance and services than individual systems (Popper, Bankes et al. 2004). Organizations are continually facing challenges to co-integrate new Component Systems (CS) and update existing systems over a while under threats, restricted budget, and uncertainty (Agarwal, Dagli et al. 2016). Both complexity and uncertainty are inherent features of infrastructure SoS, which can drive the operation of such systems away from their intended purposes (Peculis and Shirvani 2017).

It is necessary for a successful system to accurately define the interests, objectives, and requirements of its components, therefore, there are some limitations. SoS has been facing different challenges due to the configuration and functions of the systems that make SoS up. One of such challenges is managing goals to make an appropriate decision on SoS.

CS that participates in SoS arrangements might cause conflicting individual objectives among themselves. As well as, the emergence of conflicting objectives between the entire SoS, it is CS (Sage 2003).

Our objective is to have more CS within a single SoS, however, such systems must be harmonious. A conflict is an issue that occurs between two or more systems in SoS, and it represents a fundamental issue that has been extensively discussed for SoS. Such an issue might be security, pattern, classifications, or decision. Resolving the conflict does not occur through one stage but more. First, identify and detect the conflict, then diagnose to fix it appropriately (Ramsbotham, Miall et al. 2011).

Multi-systems conflicts are a generalization of a single systems conflict problem for more than one system, hence, in SoS, the more CS, the more conflicts. Several algorithms have been proposed to deal with conflicts among CS in SoS, of which most deal with any CS in SoS as a single system. In such cases, the aim is finding a conflict resolution for different systems, guaranteeing that the given solution is optimal, and managing to handle a specified number of CS.

This paper aims to find a better solution to the conflict problem. Better solutions are usually applied when SoS CS are individuals. The mission is to attract and contain more SoS and get better conflict resolution out of treating SoS CS as a joint system in clusters, and in turn, the concept of clustering, and time reduction is important from a semantic point of view. 
80 Clustering is a learning method that organises objects that are with similarities in one or more 81 features into clusters (Mokhtarpour and Stracener 2014), aiming to isolate objects with similar 82 traits and assign them into clusters. Consequently, eventually, each cluster should include 83 identical items which are different from those of other clusters.

84 Clustering algorithms are unsupervised pattern-learning algorithms (Wu, Xu et al. 2020) without 85 prior information, through creating smaller clusters with high intergroup dissimilarities and 86 intragroup similarities. A large number of these algorithms found. Hierarchical, Partitional, and

87 Bayesian are popular ones. This kind was purposefully chosen since SoS is a learned-system, and 88 unsupervised learning as a learning technique has permitted the model to work with its discovery 89 information; mainly deals with unclassified data.

90 The proposed approach aimed to adopt as many CS as possible into clusters, get such clusters to 91 form a significant SoS. Successful harmonisation of CS together in an SoS requires quick and 92 appropriate handling of conflicts.

93 There are some contributions to this paper as follows:

94 - Introduction of a new method to add more systems to SoS.

95 - Improving the handling techniques of the issues especially conflicts.

96 - Illustration of the potential of the proposed method to group more systems into clusters in 97 what resembles Sub SoSs (S-SoSs) within a large main SoS named General SoS (G-SoS).

98 - Demonstration of how the clustering method was more appropriate to cover objectives and 99 influence results, that is not in attracting more systems, but rather in reducing the number of conflicts, which has, in turn, led to more time reduction enhanced the overall results. This paper is organised as follows;

103

104

105

106

107

108

109

110

\section{1}

112

113

114

115

116

117

118

119

Part 1 covers a brief review of the conflicts in SoS, categorises all existing work into some main categories (Decision, design, security and code), orders the different approaches for solving this problem, then, in turn, displays some clustering techniques with related works. The main focus was on the method, which has been developed for use.

Part 2 presents a way to attract more system and optimize conflict resolution in SoS, explains the new algorithm (SSBFCSoS) that divided into two parts (levels). The single CS has initialised with their default (working) task, which may contain conflicts, then use k-means clustering to make them S-SoSs for the G-SoS.

\section{Background and Related Works}

The System of Systems (SoS) includes complex components and complex calculations (Gorod, White et al. 2014), with challenges of which the most significant are design, development, and decision. The detailed intricate design of SoS requires managing its CS to take advantage of it, which in turn necessitates CS interoperability rather than compatibility with diverse assets (Luzeaux 2014) (Lane and Boehm 2019), which does not usually fit the operational needs of the large SoS. CS of SoS must be able to exchange data or information among themselves, either for its purposes or for integration and fusion among them, and eventually the exchange process between the architecture of different systems, that may cause design conflicts. 
120 A survey in 2010 (Engineering 2010) explained the information and construction of SoS from an 121 engineering standpoint, combining various designs of CS that often cause conflicts. The methods 122 used often create conflicts as well, and clarify how data managed between CS and how to face 123 conflicts, through discovering another source of data in the event of a conflict. Such process presented a new conflict if no other source was available, rather than a solution to the conflict, contrary to what our research paper has provided, as in using BF to review source reliability, depending upon the trusted source system.

127 Various security priorities of multiple CS in SoS can cause conflicts (Bodeau 1994), therefore, the available security mechanisms may not meet specific SoS security requirements, leading to a more complex task of integrating and architecture SoS (Maier 1998). If a particular system has to interact securely with another CS system, its security architecture may have to deal with different authentication mechanisms, presenting a security challenge in the SoS. Some security issues of CS that make-up SoS have been discussed according to the characteristics of SoS (Madan 2015), among which is integrating the different security structures of the CS (Chiprianov, Gallon et al. 2014), which requires high characteristics of the SoS to maintain the system, and guaranteeing the services despite some security problems, in terms of networks and others.

137 The research (Ki-Aries, Faily et al. 2018) discussed an approach that coordinates the concepts of 138 SoS for analyzing security conflicts, depending on the independent collaboration of CS in SoS. The research has explained that unknown information elements have frequently impeded the risk assessment and decision-making regarding SoS. Contrary to our research, where the BF equation has been used for this, to verify that - assumed unknown - information, which has enabled us to make an appropriate decision for SoS, regardless of security conflicts either in this or other 143 research.

144 The conflict-resolution algorithm has been introduced for multi-agent systems related to relationships of different types and values (Garanina, Sidorova et al. 2015) to obtain conflict-free factors and solve ontology-based ambiguity in analyzing the natural language text. The algorithm has relied on an agent weighting conflict resolution. Contrary to our research, which has relied on the decision weight and both its accuracy and reliability, leading to more comfortable and faster processing of complex systems since it depends on addressing the conflicting data rather than the system or agent. For systems containing databases, the time factor is essential, therefore as per the research (Ahmed, Salem et al. 2015), more time may be needed to obtain data from the site, especially from a distance. The process of clustering has helped to address this challenge. The concept was reducing a large number of databases through clustering, as proposed by the research, for both distribute data and systems, reducing the number of conflicts within SoS, and reducing the mass conflict, according to the reliability estimate. It includes performance improvement and decisionmaking while saving time. assess conflicting performance, to reconcile the differences in the evaluation performance of 
160

161

162

163

164

165

166

167

168

169

170

171

172

173

174

175

176

177

178

179

180

181

182

183

184

185

186

187

188

189

190

191

192

193

194

195

196

197

198

these algorithms. These conflicts have been shown during the process of integrating the information during the decision-making process. Moreover, the proposed model can reconcile conflicting evaluation performance to reach a collective agreement in the complex decisionmaking environment.

Researchers have used the algorithm k-means to reduce the data dimensions (Idrees and Gomaa 2020), proposing a two pillars method. The first pillar is to define the consistency of traits more precisely, as in the aspect of applying clustering techniques to remove less likely features. Based on the kernel k-mean clustering algorithm, a proposed study (Muflikhah, Widodo et al. 2020) has shown that the algorithm could detect disease. Two types of datasets were extracted from DNA lysis and grouped into two groups using the kernel k-mean algorithm, then the classifier was applied to each group. When datasets aggregated, each group has significant similarities in characteristics. The block dependent classification contains more support vectors than the no-group category. Block quality also affects detection performance.

\section{Clustering and its techniques}

Clustering is a significant unsupervised learning problem, which draws references from datasets consisting of input data without specific responses, and is used as a process to find meaningful structures (Rokach and Maimon 2005).

There are no specific criteria for exemplary assembly, however, it depends on the appropriate standards for users to meet their needs. For instance, in our method, we have worked on finding representatives for Homogeneous Groups for better performance and adopted clustering to reduce working all systems together. Contrary to our approach, the previous techniques have detected conflicts on all CS of SoS, while we have used clustering to divide these CS into small clusters, leading to not only more systems attraction, but better performance and conflict resolution.

\section{Clustering methods are divided into three parts.}

a. Hierarchical: The algorithms of this technique depend on finding its clusters using precreated ones. It can be either agglomerative or divisive (Rokach and Maimon 2005). Agglomerative (bottom-up) starts with each object as a separate cluster and then merges them into larger clusters while Divisive (top-down) begins with the whole set, then divides it into smaller clusters.

b. Partitional: The algorithms here define all clusters then use the Divisive algorithms technique for grouping. It contains many techniques as Model-Based, Graph-Theoretic, and Spectral. Besides, Centroid techniques to which the k-means algorithm belongs. Clustering deals mostly with data segmentation within mega systems. Contrary to our research paper, which depends on dividing the systems within SoS rather than the data, the kmean technique has been used for the clustering process. As a partitional algorithm, it starts with a fixed number of clusters. After determining the number of clusters, the class of object $n$ is determined (Rokach and Maimon 2005), then assigned to the nearest cluster centre. The 
199

200

201

202

203

204

205

206

207

208

209

210

211

212

213

214

215

216

217

218

219

220

221

222

223

224

225

226

227

228

229

230

231

232

233

234

centres of mass $k$ are then re-estimated assuming that the above memberships are valid. This process is repeated until $n$-objects membership does not change in the last iteration.

c. Bayesian: The algorithms of this technique generate posterior distribution to collect all data partitions. It has two algorithms decision Based and Nonparametric.

\section{Smart Sematic Belief Function Clustered System of Systems method (SSBFCSOS)}

The ability of SoS to perform large tasks lies in coordinating its CS as a single system. The more the SoS can assemble more systems and do this coordination, the better. Obtaining the optimum decision from all systems while maintaining security is the goal of SoS. SoS is constructed from many CS, which conflicts among them cause SoS to be inefficient. In 2019, researchers (Younes, Ahmed et al.) have adopted a method to get the best Decision for SoS while avoiding conflicts between CS. The algorithm used was ontology with the Belief Function equation that modified to suit systems. Learning has limited SoS capacity to obtain the best results, since during running, and when CS reaches a specified number, SoS gets more time to handle conflicts. As a learned-system, it represents a challenge, so, an SSBFCSoS algorithm that depends on the clustering idea was suggested.

\section{The Handled Clustering Technique for SoS in SSBFCSoS}

Clustering is the idea for SSBFCSoS to divide CS, not data into clusters. We have primarily relied on the use of the k-means method, and a combination of this method and another agglomerative method (bottom-up). According to the used k-means method; defining each cluster according to its properties requires creating clusters that share common properties. We adopted an idea that depends on the location of the CS systems, hence each cluster contains nearby systems according to pre-specified criteria.

While in the case of partitional clustering k-means, the $k$ partitions created containing $n$ objects, each partition represents a cluster created, where $k \leq n$, attempting to divide items into clusters based on some evaluation criteria. A specific number of clusters $k$ determined by a set of centroids (initially assigning a centroid to each cluster) where $C=\left\{C_{1}, \ldots, C_{k}\right\}$. The centroid calculation for each cluster according to the mean $M_{k}$ of all the objects $N_{k}$ that belong to this cluster as in equation 1 .

$$
M_{k}=\frac{1}{N_{k}} \sum_{q=1}^{N_{k}} X(q)
$$

The members define each centre. Moreover, if the properties are incompatible, re-merge them again with any other appropriate cluster. Furthermore, this matter is repeated until each object belongs to a suitable cluster. Moreover, the stability of the clusters and their centres occurs without change. Each cluster is to be considered as Sub SoS (S-SoS) that contain systems as objects. 
235 Determining the appropriate S-SoS to belong to a particular system depends on how close the 236 traits are between systems of the same S-SoS. The primary is the close locations between 237 systems in each of S-SoS. The k-means algorithm measures the extent of similarity by

238 calculating distance. Both distance and similarity are related. Distance measure determines the 239 similarity between items and affects the shape of clusters, a smaller distance between two objects 240 results in more similarity.

241 Initialising S-SoSs is achieved by picking one point per the first S-SoS randomly as an initial

242 centroid point, then $k-1$ for the other S-SoSs; each centroid point is as far away as possible 243 from the previous centroid ones.

244 Depending on the k-means method (Solanki and Pittalia 2016), the distance is calculated 245 between each system of the S-SoS to every centroid initialised. Based on the values found, 246 systems are assigned to the centroid with the lowest distance.

247 There are four types of distances to form clusters to measure as follows;

248 Euclidean distance measure

249 Assuming that there are two systems $p$ and $q$, the Euclidean distance is a standard straight line 250 between them. The calculation is according to equation 2.

251

$$
d=\sqrt{\sum_{i=1}^{\mathrm{n}}\left(q_{i}-p_{i}\right)^{2}}
$$

252

253

254

255

256

257

258

259

260

261

262

\section{Squared Euclidean distance measure}

It is identical to the Euclidean distance measure but does not take the square root.

$$
d=\sum_{i=1}^{\mathrm{n}}\left(q_{i}-p_{i}\right)^{2}
$$

\section{Manhattan distance measure}

The absolute difference between the two systems sum computed, coordinates are shown in equation 4 . It is the distance between two systems measured along axes at right angles.

$$
d=\sum_{i=1}^{\mathrm{n}}\left|q_{x}-p_{x}\right|+\left|q_{y}-p_{y}\right|
$$

\section{Cosine distance measure}

The angle cosine between the two systems vectors is determined in the space of dimension $n$, as in equation 5, considering that $p_{i}$ and $q_{i}$ are components of vector $p$ and $q$.

$$
d=\frac{\sum_{i=0}^{\mathrm{n}-1} q_{i}-p_{i}}{\sum_{i=0}^{\mathrm{n}-1} q_{i}^{2} \times \sum_{i=0}^{\mathrm{n}-1} p_{i}{ }^{2}}
$$

263 To form clusters (S-SoSs), first, random points as S-SoS called centroids are assigned. Second, 264 assign each system to the closest S-SoS centroid by implementing a Euclidean distance (the 
265

266

267

268

269

270

271

272

273

274

275

276

277

278

279

280

281

282

283

284

285

286

287

288

289

290

291

292

293

294

295

296

297

298

299

300

301

system distance to centroid). Third, identify the new centroids by taking the average of the assigned systems points, then continue to repeat the second and third steps until convergence is achieved.

It is the first part of the SSBFCSoS algorithm. Conflicts are detected and addressed within each subsystem in the OBFSoS algorithm (Younes, Ahmed, et al.), then comes the second part of the clustering method; aggregating all S-SoSs into G-SoS, in stages that mainly depend on the number of S-SoSs.

The last part of the clustering method in SSBFCSoS belongs to the agglomerative hierarchical algorithm, through the bottom-up process. This algorithm is based on finding the distance from the closest pair of S-SoS via the distance between the two centroids $d\left(S_{-} S o S_{A B}\right)$. Assuming that the two closest clusters are S-SoS (A) and S-SoS (B), the newly formed cluster would be S-SoS (AB). Reset centroid for the new cluster, and update the distance matrix for these two clusters, giving only a distance between the new S-SoS (AB) and the other remaining clusters, then calculate the average distance between two clusters as the average distance between all systems as in equation 6 , where $d_{i k}$ represents the distance (similarity). $N\left(S_{S o S A B}\right)$ and $d\left(S_{-} S_{O} S_{C}\right)$ are numbers of systems in clusters $\mathrm{A}, \mathrm{B}$, and $\mathrm{C}$.

$$
d\left(\left(S_{-} S o S_{A B}\right)\right)_{C}=\frac{\sum_{i} \sum_{k} d_{i k}}{\left.N_{\left(S_{-} S o S_{A B}\right)}\right)^{N_{S_{-} S o S_{C}}}}
$$

Finally, detect conflicts inside the new cluster and address them using OBFSoS, then iterate these steps until all clusters merged into a single one called General SoS (G-SoS) so that all systems in G-SoS after the algorithm are terminated.

\section{Proposed Method}

The proposed method SSBFCSoS considers an enhancement of the OBFSoS method (Younes, Ahmed et al.). Both methods have adopted the concept of better decision making for SoS by addressing conflicts between CS. The proposed SSBFCSoS method consists of three phases, summarized in Fig.1.

The first phase deals with k-means, one of the clustering techniques for dividing CS into groups. Such groups use Java Format, Packages in Glest Game and StarCraft Brood War packages. The requirements are; processor Intel(R) Core (TM) i5-6200U CPU @ 2.30GHz 2.40 $\mathrm{GHz}$, RAM $8.00 \mathrm{~GB}$ and platform is 64-bit Windows 10 operating system, x64-based processor. The second phase presents the reversal of Glest and StarCraft Brood War code generating into OWL Ontologies of all systems in each cluster, together with merging the OWL ontologies for conflict detection and decision making for each S-SoS. Plus, StarCraft Brood War is converting. The third phase conflict detection of conflicts while merging the S-SoSs using another type of clustering technique; getting G-SoS better decision.

Coordinating systems with common characteristics using Ontology is easier (Gutierrez 2018), where avoiding conflicts resulting from similar systems components. The Belief Function (BF) equation assumes in the OBFSoS algorithm, which used for the SSBFCSoS method as well. BF 
302 primary use is enabling the measurement of conflict weight, and the probability of decision

303 making.

304 The algorithm is in three parts, two of which are for the clustering processes and the third is for 305 conflict resolution within each cluster summarized in Algorithm 1, which presents the pseudo-

306 code for SSBFCSoS, depending on using two clustering methods; the (k-means) method and the 307 (bottom-up) agglomerative method.

308 The first phase specifies the maximum number of desired systems $\left(s_{i}\right)$ in each cluster and the

309

310

311

312

313

314

315

316

317

318

319

320

321

322

323

324

325

326

327

328

329

330

331

332

333

334

335

336

337

338 number of desired clusters $\left(C_{l}\right)$, then, after determining the number of clusters to be divided for the systems to enter, centroids are were chosen randomly, including the rest of the systems in the clusters, hence, calculating the distance $\left(d_{s_{i}}\right)$ for connecting each system in the cluster with the closest centroids.

Calculating the distance according to the location of each system is performed by several methods. Euclidean distance measurement is the adopted method (equation $2 \& 3$ ), since it was more suitable for the homogeneous cases applied, because of the distance calculation based on the raw inputs.

The primary advantage of the Euclidean method is that the distance between any two systems is not affected by adding new systems to the analysis, which may be outliers, however, distances can be significantly affected by differences in scale between the dimensions over which distances calculated. For example, if a dimension denotes the length measured in centimetres, it is preferred not to convert it to another scale, as the resulting Euclidean can be significantly affected. Euclidean distance gives the best result (Singh, Yadav et al. 2013).

$$
J=\sum_{l=1}^{r} \sum_{i=1}^{n}\left\|s_{i}^{(l)}-C_{l}\right\|^{2}
$$

Equation 7 calculates the distance for systems, considering $r$ number of clusters, $n$ number of systems $s_{i}$ case $i$ and $C_{l}$ the centroid for cluster $l . J$ is an objective function where, $\left\|s_{i}^{(j)}-C_{l}\right\|^{2}$ represent the distance function.

After determining the inputs, all systems are considered part of a cluster (S-SoS), and the selection of the cluster depends on the centroid focal point closest to it. One system in two clusters is not allowed at the same time.

After organizing the clusters, each cluster will be addressed as a separate system, so that each cluster will resolve conflicts between the CS, obtaining the best decision and observing all the regulations and tasks related to it. All this is achieved in parallel to the clusters S-SoSs.

The second phase is the OBFSoS method role, which primarily depends on addressing conflicts and obtaining the best decision. In this method, weight is relied upon because the system relies on more data for decision-making, this means that it examines more data to make the right decision. The requirement is to obtain a system decision with the most considerable value for weight, suppose the weight values are equal in more than one system. In this case, the solution is either merging the decisions or leaving the decision to the user. 
339 The output variable of the OBFSoS method is $X_{i j}$ for optimizing decision making for SoS, the

340 weight variable $w_{i j} . X_{i j}$ represents each cluster decision. $w_{i j}$ represents the weight of this

341 decision. Each cluster as a part of General SoS (G-SoS) needs the output maintained while

342 prioritising conflict issues according to SoS requirements, putting into consideration that

343 decision-making precedes delegation decisions.

344 The third phase includes obtaining the information required from each cluster $X_{i j}$ and $w_{i j}$,

345 followed by entering into the final stage of algorithm 1, to obtain the best Decision regarding G-

346 SoS. This is the stage of comparing the obtained results from the OBFSoS algorithm, comparing

347 the effects and weights of all decisions to enable the G-SoS to obtain the optimal decision.

348 Another kind of cluster approach has been adopted to finally integrate the S-SoSs into a single

349 SoS (G-SoS). At the end of the algorithm SSBFCSoS, the two closest clusters (S-SoS) are

350 identified by calculating min distance $d(i, j)$, then merging them with the OBFSoS algorithm as

351 in $M S_{S o S}$. Then, although the weights of each S-SoS are preserved, conflicts were detected in the

352 new S-SoSs that merged; a sequence repeated until a G-SoS idealized decision is reached.

353 There is only one particular case. where there are two groups of equal weights; the two clusters

354

355

356 Case study 1 (Glest)

357 SSBFCSoS, as an algorithm represented in this paper, applies to many systems states. One of

358

359

360

361

362

363

364

365

366

367

368

369

370

371

372

373

374

375

376

377

378 these instances and the experimental setup used for our empirical analysis is described. This case has been selected and set up to match previous work (Younes, Ahmed, et al.). Ontology is useful for systems with similar components. Such systems can be called homogeneous systems. One of these homogeneous systems is gaming. The system does not just mean having software, but rather hardware and software, in addition to the human component and the communication between all of these components. This online connection can be in the case of online games, provided that every player with all these components is considered a system.

The choice was an action-based RTS (Real-Time Strategy) game called Glest (Dimitriadis 2009). Several actions in the game represent data flows, and each group of actions belongs to a category. It is impossible to deal with layers of the same name without conflict. The similarity of names between categories does not necessarily indicate the similarity of the data.

Such similarity and the resulting conflict caused by it have to be avoided, which is the ultimate role of Ontology, depending on the semantic comparison, which differentiates between the classes meanings of the same name and content with a different owner. It is also necessary for SoS to determine the most appropriate decisions. It is for SoS to perform its duties to the fullest. The systems deal with more than one stage, of which one is the resulting resolution for each cluster S-SoS. The other step is to obtain the final decision resulting from G-SoS. The goal is to determine the winner as a decision, however, the first stage of its decision is the trade-off between winning and action, as in every cluster that plays as an S-SoS has two directions. Suppose each entry system for this S-SoS plays as a separate system, the S-SoS waits for the decision of which systems to be the winner. 
379 Moreover, more than one system within this group played as multiple, here the action

380 differentiated as a decision. Any action is the right one, and the winning decisions are compared

381 to others inside the cluster. The better the number of points paid, the better the decision. The

382 points here represent the data flow; consequently, the validity of the data.

383 The faster the SoS, the better. So the individual system is handled as an intelligent agent, to save 384 time because intelligence allows the system to avoid its mistakes, repeating them, and repeated 385 conflicts.

386

387

388

389

390

391

392

393

394

395

396

397

398

399

400

401

402

403

404

405

406

407

408

409

410

411

412

413

414

415

416

417

\section{Case study 2 (StarCraft Brood War)}

The StarCraft Brood War (Dor 2014) RTS game applied the same rules as Glest. The main focus of this paper will be the final calculations of the conflict and resolution. The conflict of the Brood War represents the differences between players of each cluster of G-SoS, to evaluate the solution selection mechanism for it depending on algorithm 1 .

\section{Results and Discussion}

\section{Glest}

Both SSBFCSoS and OBFSoS have been implemented. For SSBFCSoS, the systems were divided into clusters. SSBFCSoS is very effective. The two algorithms are based on ontology and BF use. However, SSBFCSoS with this clustering still outperforms OBFSoS in many scenarios. Each attempt begins with 3 clusters, and each cluster includes 15 to 30 systems ranges. The formation of clusters depends on the location of the systems, according to system IP with a time limit of 120 seconds for both algorithms sets, which is for the OBFSoS algorithm to define conflicts between systems. For SSBFCSoS, the time for each cluster is to identify the systems involved to get conflicts. If an algorithm lacks the ability to resolve an issue within the time limit, it stops, and the failure returned. The goal here is to study the behaviour of the two algorithms for a specific number of systems.

Table 1 shows the number of conflicts generated for SSBFCSoS compared to OBFSoS averaged 70 systems. For ten attempts, in the case of SSBFCSoS, the number of conflicts for the input systems is recorded, with a success rate more than the OBFSoS algorithm.

Fig. 2 shows the conflict rate for SSBFCSoS compared to OBFSoS, which appears for 60 seconds by the algorithms for several systems. In these minor problems, SSBFCSoS is inferior in conflict rate to OBFSoS, having a slight advantage.

Different algorithms give an instance of a problem with a certain number of $s_{i}$ systems. The runtime also gives to two algorithms. Each algorithm is given 300 seconds to resolve the conflicts it encountered. SSBFCSoS divides these systems into S-SoS (clusters) and implements the algorithm associated with each sub-problem separately. Only final results for the SSBFCSoS and OBFSoS algorithms are presented.

There is a sequence in the comparison values between SSBFCSoS and OBFSoS in resolving conflicts regarding decision, design, security, and code. For the first method SSBFCSoS with 
418 each cluster value before and after resolving conflicts for all Tables 2, 3, 4, and 5, in contrast to

419 the other method OBFSoS in which the conflicts and the resolution for the whole SoS are 420 without clustering.

421 The decision is to determine which system got the best points to win the game. That is with 422 regards to systems such as an RTS game as the Glest, as a result of making the right decisions.

423 As part of the algorithm, BF is used for the validity of action sources for each system (player), 424 and using a fewer number of right actions with the same result giving the player the right to win 425 the opponent, even if their points are equal, thus, the decision to win.

426 Table 2 shows the rate of conflicts in the decisions and their solutions between SSBFCSoS and 427 OBFSoS. The number of attempts denotes the simulation attempts column. For SSBFCSoS, the 428 number of desired clusters is indicated by $C_{l}$. For the given number of $s_{i}$ systems (70), the best429 performing algorithm result is given in bold as in SSBFCSoS.

430 In Table 2, the values that resulted from applying clustering through SSBFCSoS has shown 431 better results. Attempt 5, for instance, shows the decrease of the conflicts percentage to 0.206 for 432 all the clusters in SSBFCSoS compared to 0.346 for the other method. The results have 433 progressed to reach 0.067 for SSBFCSoS compared to 0.357 for OBFSoS for the last attempt. 434 Fig.3 illustrates the rate of remaining conflicts in decisions while resolving the SSBFCSoS and 435 OBFSoS algorithms. It illustrates how SSBFCSoS encounters more conflicts on time, and is, 436 therefore, more suitable for SoS than OBFSoS.

437 Compared to OBFSoS, the SSBFCSoS algorithm curve shows that the rate of conflicts decreases 438 sequentially, as shown in Fig.3, due to the clustering of conflict handling. The system is an 439 intelligent, learned system that avoids the same conflicts encountered in earlier attempts. It 440 appears, as shown in Table 1, that applying the algorithm SSBFCSoS, the total number of 441 conflicts decreases as the number of attempts increases, so the relationship here is considered 442 inverse.

443 The system design is the general structure of the system. The parts that this system consists of 444 include units, interfaces, and data. As a system, the game also has a pattern or design.

445 Furthermore, from it, the incompatibility between the structure of two systems (two games) or 446 more, is what calls for design conflicts. Although systems are homogenous, different versions of 447 the game can cause such design conflicts. The same thing may cause code conflicts, as well. 448 The goal is to make the right decision to determine the winning player and also collect points. In 449 case there are design conflicts between two or more systems, the right decision cannot be made, 450 because of the incompatibility between the design of the two systems. According to the rules, to 451 determine the best work, the environment should be equal, since incompatible environments lead 452 to design conflicts, depending on the different versions of the game. If Algorithm is used, 453 SSBFCSoS determines the compatibility. BF also returns to the game source to verify this. 454 Table 3 displays the rate of conflicts in design with its solutions between SSBFCSoS and 455 OBFSoS. For example, in the last attempt, conflicts in the design percentage reached a ratio of 4560.067 in SSBFCSoS compared to 0.243 in OBFSoS, which is considered a good result, as shown 457 in Table 3 that almost all the conflicts in two clusters were dealt with against one conflict in the 
458 last cluster concerning method SSBFCSoS. While OBFSoS needed a longer time to reach the 459 same results. The clustering process dealt with conflicts in parallel, thus it consumed less time 460 than without it.

461 Fig.4 illustrates the rate of remaining conflicts in design after they encountered, during the 462 process of resolving the SSBFCSoS and OBFSoS algorithms. It describes how SSBFCSoS faces 463 more conflicts than OBFSoS in a limited time.

464 In Table 4, the results of the comparison of SSBFCSoS and OBFSoS shown in the code 465 conflicts. In addition to clarifying the clusters, each cluster is presented separately before and

466

467

468

469

470

471

472

473

474

475

476

477

478

479

480

481

482

483

484

485

486

487

488

489

490

491

492

493

494

495

496

497 after applying the SSBFCSoS algorithm. SSBFCSoS was able to face conflicts almost double the other method. Whereas for SSBFCSoS, the conflict ratio has decreased to about 0.067 compared to 0.114 for OBFSoS. Either way, it consumed about the same time and could be less. Fig. 5 shows the sequence of results for both algorithms to resolve conflicts in code. The higher the number of attempts, the better the outcome for each algorithm, however, algorithm SSBFCSoS still produces significantly better results as in the figure.

In regards to priority for each system, it may be a security priority. Some systems place security at the top of their interests, according to their goals. In this paper, the systems are gaming systems. Moreover, the game has an online version, therefore, security becomes essential. The importance of security lies in its influence on the decision of our SoS. So, it is considered working for security if it affects the decision because getting a better decision is the first and primary goal.

The score has to be confirmed to determine who won, which is why, in this case, not all players can enter the game until they pass the registration rules. That is to achieve the security requirement. For that, Table 5 records the evolution in the number of conflicts in security. In Table 5, SSBFCSoS achieved good results in facing security conflicts as much as possible. The conflicts ratio was at attempt 7 was about 0.080 and 0.126 for OBFSoS for the same attempt. Fig.6 shows the rate of development of security conflicts after applying methods, showing satisfactory results by the SSBFCSoS in comparison to OBFSoS.

Table 6 shows the conflict rate resolved by the algorithms, and the time takes for each algorithm to obtain these solutions. We have reached a better SSBFCSoS algorithm to get good results, which consumes a shorter time. As shown in Table 6, the first attempt, the resolving conflicts percentage for the SSBFCSoS has reached 0.296 within approximately 281 seconds, while OBFSoS achieved 0.076 within 300 seconds. As for the last attempt, the rate of resolving conflicts has reached 0.733 for the SSBFCSoS within 113 seconds, while OBFSoS has achieved about 0.171 within 0.276 seconds.

These rates show in Fig.7, which reflects the rate of the conflicts resolved.

Fig. 8 shows a comparison of the time taken for each algorithm to obtain solutions, proving that the SSBFCSoS algorithm is the best in terms of time consumption.

\section{StarCraft Brood War}

To evaluate the StarCraft Brood War, the conflict resolution rates in the cases under consideration generated by SSBFCSoS and OBFSoS are calculated and listed in Table 7. For 
498 decision conflicts appearance as in attempt 9, the SSBFCSoS has reached 0.250 compared to 4990.394 by OBFSoS. For design conflicts appearance as in attempt 8, the SSBFCSoS has reached 5000.250 compared to 0.287 by OBFSoS. As for security conflicts in the same attempt, SSBFCSoS 501 has reached 0.167 compared to 0.204 by OBFSoS.

502 Table 8, shows that SSBFCSoS has achieved superiority over OBFSoS in terms of conflict 503 resolution in SoS.

504 Table 8 presents the number of conflicts generated for SSBFCSoS compared to OBFSoS with an 505 average of 100 systems for G-SoS. SSBFCSoS divides these systems into 3 clusters (S-SoSs), 506 with the number of systems ranging from 25 to 36 per cluster. Each algorithm is allowed 400 507 seconds to resolve the conflicts encountered.

508 Table 8 shows only the final result for the three merged clusters in decision, design, code and 509 security conflicts. At attempt 8, the percentage of conflicts after applying SSBFCSoS has 510 reached 0.208 compared to 0.389 for OBFSoS in decision conflicts. In design, conflicts have 511 reached 0.185 for SSBFCSoS compared to 0.242 for OBFSoS in attempt 7. In attempt 9, the 512 SSBFCSoS conflict ratio has reached 0.100 compared to 0.154 for OBFSoS in terms of security, 513 and the code conflicts ratio has reached 0.100 for SSBFCSoS compared to 0.163 for OBFSoS.

514 Fig.9 shows the improvement in the conflicts rate in decisions for SoS achieved by SSBFCSoS 515 in comparison to that achieved by OBFSoS.

516 Fig.10 shows that the conflicts in the design reduced by using SSBFCSoS better than OBFSoS. 517 Fig.11 with almost the same conflict rate for SSBFCSoS and OBFSoS. However, SSBFCSoS 518 can resolve more conflicts than OBFSoS.

519 The curve in Fig.12 illustrates the code conflicts of the two methods.

520 Table 9 shows the rate of addressing conflicts of the SoS in the ratio of both SSBFCSoS and 521 OBFSoS along with the time taken. In attempt 7, the percentage of solutions achieved by 522 SSBFCSoS has reached 0.333 within 127 seconds compared to a ratio of 0.70 within 317 523 seconds for OBFSoS.

524 Fig.13 shows the extent to which SSBFCSoS was better than OBFSoS in terms of addressing 525 conflicts, and its rate has reached $67 \%$ in attempt 10 compared to $17 \%$ for OBFSoS.

526 Fig.14 Compares the time spent for each method, showing the advantage of SSBFCSoS in saving 527 time at a rate of more than three times the time consumed by OBFSoS.

528 Each of the two systems revealed the number of conflicts. The results indicated the progress of 529 SSBFCSoS, which has reached this success thanks to the division of inputs from the systems to 530 fall under the name clusters S-SoS. It also shows an apparent effect on the proper use of time. 531 Focusing on time is essential in exceptional cases, such as the systems to which it was applied.

\section{Comparing the two methods SSBFCSoS and OBFSoS}

533 Experience clearly shows that there is no established law for making a better Decision for SoS. 534 However, the performance of each of the applied algorithms depends on the features of the 535 conflicts. 
536 At the beginning of the application, a certain number - three - of clusters are identified, with each 537 cluster containing 20 to 35 systems, ultimately reaching about 70 or 100 systems. Time is set to 538 approximately 120 seconds to identify conflicts, and about 300 seconds to address those 539 conflicts. These features can be of conflict type and number of systems that have conflict at the 540 same time, in addition to the number of conflicts encountered during the solution process.

541 Each algorithm has its method depending on the case. Nevertheless, through research, a method

542 of evaluating the performance of each algorithm has been proposed. So, we present the following 543 general trends that we observed:

544

545

546

547

548

549

550

551

552

553

554

555

556

557

558

559

560

561

562

563

564

565

566

567

568

569

570

571

572

573

- The algorithm SSBFCSoS outperforms the other algorithm in most cases with an unlimited number of systems.

- The longer the time is taken by algorithm OBFSoS, the better-achieved results, but to a limited number of systems only.

- As for the clusters in algorithm SSBFCSoS, the system must not be part of more than one cluster simultaneously.

- The game running online, allows the system to exit and enter more than once. It could create a repeated conflict, especially in security.

- Although the algorithm system SSBFCSoS is a learned system. However, it can seriously increase in some attempts over others in conflicts for the previously mentioned reasons.

- Continuous attempts with algorithm SSBFCSoS reduce the number of conflicts and the time taken to resolve conflicts.

- There is a constant conflict despite repeated attempts, which requires user intervention to get the appropriate and better decision. Therefore, a significant percentage of about $20 \%$ of the unresolved conflicts was required for user intervention.

Fig. 15 shows the number of systems and clusters and the conflict resolution rate. The number of clusters was set proportional to the number of systems, creating a direct relationship; the more systems and clusters, the more satisfactory the results. Should we let the system limit the number of clusters? If we leave it open, it is possible to create semi-empty clusters containing only two or three systems. So, we tried to define the systems. During implementation, we found that if the average number of systems within each cluster was $10 \%$ of the total number of systems, the results were very appropriate, and the time was right. Also, if the average systems for each cluster are 25-30, the results are perfect.

Also, we found a second situation during implementation where two or more systems (players) play with the multiplayer system, as in the two systems produce one combined decision. First, we need to compare procedures by OBFSoS algorithm, then compare their decisions. We have two parts to compare the procedures for obtaining the highest points first and determining the best procedure for obtaining higher points for their group, then compare the decisions to get an ideal decision. And then they entered as part of the S-SoS and so on.

Comparing clustering methods k-means and agglomerative (bottom-up)

Peer) Comput. Sci. reviewing PDF | (CS-2020:10:54200:3:0:NEW 5 Mar 2021) 
574 We made a statistic that compared two clustering algorithms, namely k-means and agglomerative 575 (bottom-up). The two algorithms were compared in the second case study; StarCraft Brood War.

576 We made this statistic on different numbers of systems, as shown in Table 10. We were

577 beginning with fewer systems, ending with more. We recorded the values after seven attempts.

578 Concerning the algorithm k-means, we specified the number of clusters as 5 clusters. Contrary to

579 k-means, the number of clusters in agglomerative (bottom-up) is not determined, but each system

580 is considered as a separate cluster until several clusters formed. The same distance measurement

581 is used for the k-means.

582 In Table 10 for case 7 with 110 systems as inputs, k-means achieved a conflict resolution rate of

583 about $90 \%$ compared to about $69 \%$ for the agglomerative (bottom-up). These results took about

584279 seconds for the k-means compared to 790 seconds for the other one.

585 Fig.16 clarifies the comparison curve between the two-clustering methods k-means and

586 agglomerative (bottom-up) so that k-means showed excellent results better than the other

587 method.

588 Fig. 17 gave a curve for the time consumed for each method to obtain these results, showing a

589 significant difference in the time taken between the two methods, with k-means saving time

590 while preserving its results better than the other method.

591 Generally, the k-means algorithm is easy to implement and more suitable with big data such as

592 SoS (Nasraoui and N'Cir 2019).

593 Comparison between the k-means algorithm and some clustering algorithms

594 The choice of the k-mean algorithm as a clustering technique for optimizing SSBFCSoS is

595 supported. SoS features are a combination of some IoT features and some of the big data,

596 therefore, we need a clustering algorithm with the following features for the fusion into

597 SSBFCSoS:

598 - Dealing with huge datasets such as k-means, CURE, BIRCH and CHAMELEON (Sanse

599 and Sharma 2015), STING (Oyelade, Isewon et al. 2016), Agglomerative, and

600 HASTREAM (Nasraoui and N'Cir 2019). We display the data size criteria in the second

$601 \quad$ column Table 11.

602

603

604

605

606

- Low time consumption. K-means is a very fast algorithm reducing the overall consumed time (Nasraoui and N'Cir 2019).

- Not requiring special hardware as all chosen algorithms in Table 11.

- Scalability, the k-means algorithm is at the forefront of scalability as for SoS (Sholla, Kaur et al. 2017).

607

608

- Accepting overlapping clusters, which is the effect on conflict solution. k-means is one of

609

610

611

612 the algorithms that do not accept overlap between clusters (Khanmohammadi, Adibeig et al. 2017) as well as OPTICS (Mirzaie, Barani et al. 2015), HASTREAM and Liar Tree (Nasraoui and N'Cir 2019), While algorithms C-means (He, Guo et al. 2018), SOM (Sarlin and Eklund 2011) and K-Medoids (Arora and Varshney 2016) accept overlapping. This comparison is in column four, Table 11. 
613

614

615

616

617

618

619

620

621

622

\section{Conclusions}

624

625

626

627

628

629

630

631

632

633

634

635

636

637

638

639

640

641

642

643

644

645

646

647

648 Two types of clustering algorithms have been tried; k-means and agglomerative (bottom-up).

649 The algorithm k-means was adopted for SSBFCSoS for its better results, not only in terms of 650

Increasing the SoS capacity to accommodate more systems results in some issues among the SoS Components systems. Conflict is one of these issues. This paper focused on how to enhance the speed of detection and solution of conflicts that may arise while integrating new systems into an existing SoS.

We presented a method based on the use of the k-means clustering technique. Each cluster contains nearby systems according to pre-specified criteria. We can consider each cluster a Sub SoS (S-SoS) which in turn form the major SoS. We proposed the Smart Semantic Belief Function Clustered System of Systems (SSBFCSoS) which is an enhancement of the Ontology Belief Function System of Systems (OBFSoS). The proposed method proved the ability to detect and solve conflicts.

In order to test the applicability of the SSBFCSoS and compare its performance with other approaches, two well-known datasets were employed. They are (Glest \& StarCraft Brood War). With each dataset, 15 test cases were examined. Considering that each testing was after every ten attempts of learning. To evaluate the proposed method, we applied it to homogeneous systems cases. The Glest game for a sample of about 70 systems divided into three clusters (S-SoSs). StarCraft Brood War was selected for a sample of about 100 systems divided into three clusters (S-SoSs). Furthermore, we have picked these cases specifically for the application because we intend to use homogeneous states.

Using clustering techniques showed better performance ratios. The proposed method (SSBFCSoS) demonstrated the time-reducing effect of SoS activities with the early detection of conflicts to be dealt with. We adopted an essential type of conflict, which is the struggle to obtain the optimal Decision for SoS. As a learned system, it avoids similar conflicts during attempts. It also helped to improve results and allowed us to deal with more CS in SoS with saving much time.

Peer) Comput. Sci. reviewing PDF | (CS-2020:10:54200:3:0:NEW 5 Mar 2021) 
651 In many cases, clustering results enabled the SSBFCSoS to check the conflict state while still

652 handling conflict optimally. We achieved, on average, $89 \%$ in solving the conflict compared to

$65377 \%$ of the other well-known approaches. Moreover, it showed an acceleration of up to

654 proportionality over previous approaches for about $16 \%$ in solving conflicts as well. It also

655 reduces the frequency of the same conflicts by approximately $23 \%$ better than the other method,

656 not only in the same cluster but even while combining different clusters. Eventually; the positive

657 effect of the clustering process has appeared, the number of Component Systems (CS) has almost

658 tripled compared to other methods while preserving integration and conflict resolution.

\section{Future Work}

660 The evaluation of this approach appears promising, but scalability issues remain to addressed.

661 Consequently, future business trends should include developing more specific technologies to

662 define conflict for larger quantities of systems and rules of conflicts. Besides, dealing with

663 heterogeneous systems such as those found in smart cities. It can also be used as the

664 classification method developed for different types of conflicts to provide orderly results to the

665 user.

666 We used the k-means technique which depends on identifying the desired number of clusters

667 before initiating the clustering process. We found that when some new systems are added to the

668 already existing SoS, the system may belong to an S-SoS far from the one closest to it. That

669 deviates us from the idea that we relied on in classification to some extent. This would prompt us

670 to try different clustering techniques in the future.

671 Finally, the application on different numbers of systems within more than one method of

672 clustering shows another issue; which is how to reach the ideal number of systems within each

673 cluster.

674 Implementation showed that when the number of systems within one cluster reaches a specific

675 number, the SoS becomes ideal in achieving its goals, which is evidently, the ideal number of

676 clusters to achieve the SoS goal. This opens up another avenue for research on the same issue for

677 future work. Besides, we will put a competitive study with Artificial Intelligence clustering

678 techniques for the generalization of our proposed method (SSBFCSoS) in the future as well.

679

\section{References}

681 Agarwal, S., C. H. Dagli and L. E. Pape (2016). Computational intelligence based complex

682 adaptive system-of-system architecture evolution strategy. Complex Systems Design \&

683 Management, Springer: 119-132.

684 Ahmed, H., R. Salem and S. a. S. Saleh (2015). "Clustering Algorithm for Distributed Real-Time

685 Database sites." IJCI. International Journal of Computers and Information 4(1): 11-20.

686 Arora, P. and S. Varshney (2016). "Analysis of k-means and k-medoids algorithm for big data."

687 Procedia Computer Science 78: 507-512.

688 Bangui, H., M. Ge and B. Buhnova (2018). Exploring Big Data Clustering Algorithms for Internet

689 of Things Applications. IoTBDS. 
690 Bodeau, D. J. (1994). System-of-systems security engineering. Tenth Annual Computer

691

692

693

694

695

696

697

698

699

700

701

702

703

704

705

706

707

708

709

710

711

712

713

714

715

716

717

718

719

720

721

722

723

724

725

726

727

728

729

730

731

732

733

734

735

736

737

738

Security Applications Conference, IEEE.

Boehm, B. and J. A. Lane (2007). "Using the incremental commitment model to integrate system acquisition, systems engineering, and software engineering." CrossTalk 19(10): 4-9.

Chiprianov, V., L. Gallon, M. Munier, P. Aniorte and V. Lalanne (2014). "The systems-ofsystems challenge in security engineering." Groupement De Recherche CNRS du Génie de la Programmation et du Logiciel: 163.

Dimitriadis, V. K. (2009). "Reinforcement Learning in Real Time Strategy Games Case Study on the Free Software Game Glest." Department of Electronic and Computer Engineering Technical University of Crete. China.

Dor, S. (2014). "The heuristic circle of real-time strategy process: A starcraft: Brood war case study." Game Studies 14(1).

Engineering, D. o. S. (2010). "Systems Engineering Guide for System of

Systems." Defense Research and Engineering, Pentagon, Washington, D.C. 20301-3040: 17.

Garanina, N., E. Sidorova and S. Anokhin (2015). Conflict resolution in multi-agent systems with typed connections for ontology population. International Andrei Ershov Memorial Conference on Perspectives of System Informatics, Springer.

Gorod, A., B. E. White, V. Ireland, S. J. Gandhi and B. Sauser (2014). Case studies in system of systems, enterprise systems, and complex systems engineering, CRC Press.

Gutierrez, R. (2018). A Requirement Ontology To Guide The Analysis Of System Life Cycle Processes, Concordia University.

He, X., K. Guo, Q. Liao and Q. Yan (2018). Overlapping Community Detection Algorithm Based on Spectral and Fuzzy C-Means Clustering. CCF Conference on Computer Supported Cooperative Work and Social Computing, Springer.

Idrees, A. M. and W. H. Gomaa (2020) "A Proposed Method for Minimizing Mining Tasks' Data Dimensionality."

Khanmohammadi, S., N. Adibeig and S. Shanehbandy (2017). "An improved overlapping kmeans clustering method for medical applications." Expert Systems with Applications 67: 12-18. Ki-Aries, D., S. Faily, H. Dogan and C. Williams (2018). Assessing system of systems security risk and requirements with OASoSIS. 2018 IEEE 5th International Workshop on Evolving Security \& Privacy Requirements Engineering (ESPRE), IEEE.

Lane, J. A. and B. Boehm (2019). Systems of Systems Thinking. Systems Engineering in Context, Springer: 553-564.

Luzeaux, D. (2014). SoS and large-scale complex systems architecting. Complex Systems Design \& Management, Springer: 39-49.

Madan, B. B. (2015). "System of Systems Security." Modeling and Simulation Support for System of Systems Engineering Applications: 565.

Maier, M. W. (1998). "Architecting principles for systems-of-systems." Systems Engineering:

The Journal of the International Council on Systems Engineering 1(4): 267-284.

Mirzaie, M., A. Barani, N. Nematbakkhsh and M. Beigi (2015). "OverDBC: A new density-based clustering method with the ability of detecting overlapped clusters from gene expression data." Intelligent Data Analysis 19(6): 1311-1321.

Mokhtarpour, B. and J. Stracener (2014). Application of a clustering technique in identifying the "best" System of Systems (SoS) during development. 2014 IEEE International Conference on Systems, Man, and Cybernetics (SMC), IEEE.

Muflikhah, L., W. Widodo, W. F. Mahmudy and S. Solimun (2020) "A Support Vector Machine Based on Kernel K-Means for Detecting the Liver Cancer Disease."

Nasraoui, O. and C.-E. B. N'Cir (2019). Clustering Methods for Big Data Analytics. Techniques, Toolboxes and Applications, Springer: 192.

Peer) Comput. Sci. reviewing PDF | (CS-2020:10:54200:3:0:NEW 5 Mar 2021) 
739 Oyelade, J., I. Isewon, F. Oladipupo, O. Aromolaran, E. Uwoghiren, F. Ameh, M. Achas and E.

740 Adebiyi (2016). "Clustering algorithms: their application to gene expression data." Bioinformatics

741 and Biology insights 10: BBI. S38316.

742 Peculis, R. and F. Shirvani (2017). "Infrastructure system of systems integrity."

743 Popper, S. W., S. C. Bankes, R. Callaway and D. DeLaurentis (2004). "System of systems

744 symposium: Report on a summer conversation." Potomac Institute for Policy Studies, Arlington,

745 VA 320.

746 Ramsbotham, O., H. Miall and T. Woodhouse (2011). Contemporary conflict resolution, Polity.

747 Robinson, W. N., S. D. Pawlowski and V. Volkov (2003). "Requirements interaction

748 management." ACM Computing Surveys (CSUR) 35(2): 132-190.

749 Rokach, L. and O. Maimon (2005). Clustering methods. Data mining and knowledge discovery

750 handbook, Springer: 321-352.

751 Sage, A. P. (2003). Conflict and risk management in complex system of systems issues.

752 SMC'03 Conference Proceedings. 2003 IEEE International Conference on Systems, Man and

753 Cybernetics. Conference Theme-System Security and Assurance (Cat. No. 03CH37483), IEEE.

754 Sanse, K. and M. Sharma (2015). "Clustering methods for Big data analysis." International

755 Journal of Advanced Research in Computer Engineering \& Technology (IJARCET) 4(3).

756 Sarlin, P. and T. Eklund (2011). Fuzzy clustering of the self-organizing map: some applications

757 on financial time series. International Workshop on Self-Organizing Maps, Springer.

758 Sholla, S., S. Kaur, G. R. Begh, R. N. Mir and M. A. Chishti (2017). "Clustering Internet of

759 Things: A Review." Journal of Science and Technology: Issue on Information and

760 Communications Technology 3(2): 21-27.

761 Singh, A., A. Yadav and A. Rana (2013). "K-means with Three different Distance Metrics."

762 International Journal of Computer Applications 67(10).

763 Solanki, K. and P. Pittalia (2016). "Review of face recognition techniques." International Journal

764 of Computer Applications 133(12): 20-24.

765 Spanoudakis, G. and A. Zisman (2001). Inconsistency management in software engineering:

766 Survey and open research issues. Handbook of Software Engineering and Knowledge

767 Engineering: Volume I: Fundamentals, World Scientific: 329-380.

768 Viana, T., A. Zisman and A. K. Bandara (2017). Identifying conflicting requirements in systems

769 of systems. 2017 IEEE 25th International Requirements Engineering Conference (RE), IEEE.

770 Wu, W., Z. Xu, G. Kou and Y. Shi (2020). "Decision-Making Support for the Evaluation of

771 Clustering Algorithms Based on MCDM." Complexity 2020.

772 Younes, H. R., A. S. E. Ahmed and E. K. Elsayed "Enhancement Belief Function with Ontology

773 to Solve the Conflict for the System of Systems."

774 


\section{Box 1 (on next page)}

Algorithm 1 Smart Sematic Belief Function Clustered System of Systems (SSBFCSoS) 


\section{Input \\ $s_{i}\left(s_{1}, s_{2}, \ldots, s_{n}\right)(i=2, \ldots ., n) / /$ Number of desired systems in each cluster \\ $C_{l}\left(c_{1}, c_{2}, \ldots, c_{r}\right)(l=1,2, \ldots ., r) / /$ Number of desired clusters}

2. $C=0 / /$ Cluster Index

3. ClusteredSystems $=0$

4. for (each unclustered $s_{i}$ )

5. $s_{i} \leftarrow$ RandomSystems $(i, n) / /$ Choose system at random

6. $C_{l} \leftarrow s_{i} / /$ Make this random system as first cluster center

7. $C++/ /$ initialize new cluster

8. ClusteredSystems ++

9. for $l \leftarrow 2 \ldots r$ do // loop over the rest of the centres

10. for $i \leftarrow 1$...n do // loop over the systems

11. $d_{s_{i}} \leftarrow \min _{i<l}\left\|\operatorname{sqr}\left(d_{s_{i}}-d_{C_{l_{\text {cent }}}}\right)\right\|^{\wedge} 2 / /$ Compute the distance to the closest centre

12. Add $s_{i}$ to the cluster $C_{l} / /$ Systems enter the closest centroid Clusters

13. end for

14. for $i \leftarrow 1$...n do // loop over the systems again

15. $c_{l} \leftarrow d^{2}{ }_{n} / \sum_{i} d_{i}^{2}$.// Compute a distribution proportional

16. end for

17. Recalculate centroids

18. $C_{r} \leftarrow s_{n} / /$ Draw systems to each cluster from distance

19. end for

20. if (ClusteredSystems $=s_{n}$ ) break; // Clustering is complete

21. Implement OBFSoS Algorithm between generated clusters results // OSoS

22. for $l=1: r$ do // Clusters

23. Implement OBFSoS for each cluster

24. end for

25. Get output from OBFSoS $X_{i j}, w_{i j} / /$ Decision \& Weight

26. $\left(X_{i j}\right)_{n e w} \leftarrow X_{i j},\left(w_{i j}\right)_{\text {new }} \leftarrow w_{i j},\left(X_{i j}\right)_{\text {old }} \leftarrow 0$, $\left(w_{i j}\right)_{\text {old }} \leftarrow 0$

27. for $x=1,3,4, \ldots ., r$ do $/ /$ General SoS

28. if $\left(w_{i j}\right)_{\text {new }}>\left(w_{i j}\right)_{\text {old }}$

29. $G \operatorname{SoS} X_{i j} \leftarrow\left(X_{i j}\right)_{\text {new }}$

30. $\left(X_{i j}\right)_{\text {old }} \leftarrow\left(X_{i j}\right)_{\text {new }}$

31. else if $\left(w_{i j}\right)_{\text {old }}>\left(w_{i j}\right)_{\text {new }}$

32. $G S o S X_{i j} \leftarrow\left(X_{i j}\right)_{\text {old }}$

33. else // ::: $\left(w_{i j}\right)_{\text {old }}=\left(w_{i j}\right)_{\text {new }}$

34. $G S o S X_{i j} \leftarrow\left(X_{i j}\right)_{\text {old }} \bowtie \leftarrow\left(X_{i j}\right)_{\text {new }}$

35. $\left(X_{i j}\right)_{\text {old }} \leftarrow\left(X_{i j}\right)_{\text {new }}$

36. end for

37. A set of $n$ systems for each cluster

38. for $i=1: n d o$

39. for $j=1: n d o$

40. $d(i, j)=$ distance function $(c(i), c(j))$

41. end for

42. $\operatorname{pair}(i)=\min (d(i, j=1: n))$ 
43. end for

44. for $k=1: n$ do

45. $M S_{\text {SoS }}=\operatorname{pair}(i)$

46. Get min distance $\left(M S_{S o S}, S_{S o S}\right)$

47. $M S o S=$ merge $\left(M S_{S o S} \& S_{S o S}\right)$

48. back to step 13

49. end for

50. $G S o S=M S o S$

Output $G_{S o S} X_{i j}$

1 


\section{Table $\mathbf{1}$ (on next page)}

Glest \# of conflicts rate generated for SSBFCSoS vs. OBFSoS 


\begin{tabular}{c|c|c}
\hline \multirow{2}{*}{$\begin{array}{c}\text { Glest } \\
\text { simulation attempts }\end{array}$} & \multicolumn{2}{|c}{ \# of conflicts } \\
\cline { 2 - 3 } & SSBFCSoS & OBFSoS \\
\hline $\mathbf{1}$ & 54 & 131 \\
\hline $\mathbf{2}$ & 47 & 125 \\
\hline $\mathbf{3}$ & 45 & 120 \\
\hline $\mathbf{4}$ & 38 & 115 \\
\hline $\mathbf{5}$ & 34 & 107 \\
\hline $\mathbf{6}$ & 30 & 102 \\
\hline $\mathbf{7}$ & 25 & 95 \\
\hline $\mathbf{8}$ & 19 & 88 \\
\hline $\mathbf{9}$ & 17 & 82 \\
\hline $\mathbf{1 0}$ & 15 & 70 \\
\hline
\end{tabular}




\section{Table 2 (on next page)}

Glest Conflicts rate in decisions generated before and after implementing the SSBFCSoS vs. OBFSoS algorithms 


\begin{tabular}{|c|c|c|c|c|c|c|c|c|c|c|}
\hline \multirow{5}{*}{$\begin{array}{c}\text { Glest } \\
\text { Simulation } \\
\text { Attempts }\end{array}$} & \multicolumn{8}{|c|}{ SSBFCSoS } & \multicolumn{2}{|c|}{ OBFSoS } \\
\hline & \multicolumn{10}{|c|}{ Conflicts rate for systems $n=70$, Run time $=300$ seconds } \\
\hline & \multicolumn{4}{|c|}{ Before Method } & \multicolumn{4}{|c|}{ After Method } & \multirow{3}{*}{$\begin{array}{c}\# \\
\text { conflicts } \\
\text { Before } \\
\text { Method }\end{array}$} & \multirow{3}{*}{$\begin{array}{c}\# \\
\text { conflicts } \\
\text { After } \\
\text { Method }\end{array}$} \\
\hline & \multicolumn{3}{|c|}{$\begin{array}{l}\text { clusters conflicts } \\
\text { in decision }\end{array}$} & \multirow{2}{*}{$\begin{array}{c}\# \\
\text { conflicts }\end{array}$} & \multicolumn{3}{|c|}{$\begin{array}{l}\text { clusters conflicts } \\
\text { in decision }\end{array}$} & \multirow{2}{*}{$\begin{array}{c}\# \\
\text { conflicts }\end{array}$} & & \\
\hline & C1 & C2 & C3 & & C1 & C2 & C3 & & & \\
\hline 1 & 0.250 & 0.350 & 0.400 & 0.370 & 0.200 & 0.300 & 0.300 & 0.296 & 0.397 & 0.366 \\
\hline 2 & 0.250 & 0.375 & 0.375 & 0.340 & 0.188 & 0.313 & 0.375 & 0.298 & 0.408 & 0.360 \\
\hline 3 & 0.267 & 0.400 & 0.333 & 0.333 & 0.133 & 0.267 & 0.267 & 0.222 & 0.417 & 0.350 \\
\hline 4 & 0.333 & 0.333 & 0.333 & 0.316 & 0.167 & 0.250 & 0.250 & 0.211 & 0.417 & 0.339 \\
\hline 5 & 0.273 & 0.273 & 0.455 & 0.324 & 0.091 & 0.182 & 0.364 & 0.206 & 0.411 & 0.346 \\
\hline 6 & 0.222 & 0.222 & 0.556 & 0.300 & 0.111 & 0.111 & 0.333 & 0.133 & 0.422 & 0.343 \\
\hline 7 & 0.500 & 0.167 & 0.333 & 0.240 & 0.333 & 0.167 & 0.167 & 0.160 & 0.421 & 0.326 \\
\hline 8 & 0.250 & 0.250 & 0.500 & 0.211 & 0.250 & 0.000 & 0.250 & 0.105 & 0.398 & 0.341 \\
\hline 9 & 0.667 & 0.000 & 0.333 & 0.176 & 0.333 & 0.000 & 0.000 & 0.059 & 0.415 & 0.341 \\
\hline 10 & 0.333 & 0.333 & 0.333 & 0.200 & 0.000 & 0.000 & 0.333 & 0.067 & 0.414 & 0.357 \\
\hline
\end{tabular}




\section{Table 3(on next page)}

Glest Conflicts rate in a design generated before and after implementing the SSBFCSoS vs. OBFSoS algorithms 


\begin{tabular}{|c|c|c|c|c|c|c|c|c|c|c|}
\hline \multirow{5}{*}{$\begin{array}{c}\text { Glest } \\
\text { Simulation } \\
\text { Attempts }\end{array}$} & \multicolumn{8}{|c|}{ SSBFCSoS } & \multicolumn{2}{|c|}{ OBFSoS } \\
\hline & \multicolumn{10}{|c|}{ Conflicts rate for systems $n=70$, Run time $=300$ seconds } \\
\hline & \multicolumn{4}{|c|}{ Before Method } & \multicolumn{4}{|c|}{ After Method } & \multirow{3}{*}{$\begin{array}{c}\# \\
\text { conflicts } \\
\text { Before } \\
\text { Method }\end{array}$} & \multirow{3}{*}{$\begin{array}{c}\# \\
\text { conflicts } \\
\text { After } \\
\text { Method }\end{array}$} \\
\hline & \multicolumn{3}{|c|}{$\begin{array}{l}\text { clusters conflicts } \\
\text { in design }\end{array}$} & \multirow{2}{*}{$\begin{array}{c}\# \\
\text { conflicts }\end{array}$} & \multicolumn{3}{|c|}{$\begin{array}{l}\text { clusters conflicts } \\
\text { in design }\end{array}$} & \multirow{2}{*}{$\begin{array}{c}\# \\
\text { conflicts }\end{array}$} & & \\
\hline & C1 & C2 & C3 & & C1 & C2 & C3 & & & \\
\hline 1 & 0.308 & 0.231 & 0.462 & 0.241 & 0.231 & 0.154 & 0.308 & 0.167 & 0.282 & 0.260 \\
\hline 2 & 0.273 & 0.273 & 0.455 & 0.234 & 0.182 & 0.182 & 0.364 & 0.170 & 0.280 & 0.264 \\
\hline 3 & 0.250 & 0.417 & 0.333 & 0.267 & 0.167 & 0.250 & 0.250 & 0.178 & 0.275 & 0.250 \\
\hline 4 & 0.300 & 0.400 & 0.300 & 0.263 & 0.200 & 0.200 & 0.200 & 0.158 & 0.278 & 0.243 \\
\hline 5 & 0.222 & 0.444 & 0.333 & 0.265 & 0.111 & 0.333 & 0.111 & 0.147 & 0.290 & 0.252 \\
\hline 6 & 0.375 & 0.375 & 0.250 & 0.267 & 0.375 & 0.125 & 0.125 & 0.167 & 0.284 & 0.235 \\
\hline 7 & 0.286 & 0.571 & 0.143 & 0.280 & 0.143 & 0.286 & 0.000 & 0.120 & 0.305 & 0.242 \\
\hline 8 & 0.000 & 0.333 & 0.667 & 0.316 & 0.000 & 0.167 & 0.167 & 0.105 & 0.295 & 0.250 \\
\hline 9 & 0.400 & 0.600 & 0.000 & 0.294 & 0.200 & 0.200 & 0.000 & 0.118 & 0.293 & 0.244 \\
\hline 10 & 0.250 & 0.500 & 0.250 & 0.267 & 0.000 & 0.250 & 0.000 & 0.067 & 0.286 & 0.243 \\
\hline
\end{tabular}




\section{Table 4 (on next page)}

Glest Conflicts rate in code generated before and after implementing the SSBFCSOS vs. OBFSOS algorithms 


\begin{tabular}{|c|c|c|c|c|c|c|c|c|c|c|}
\hline \multirow{5}{*}{$\begin{array}{c}\text { Glest } \\
\text { Simulation } \\
\text { Attempts }\end{array}$} & \multicolumn{8}{|c|}{ SSBFCSoS } & \multicolumn{2}{|c|}{ OBFSoS } \\
\hline & \multicolumn{10}{|c|}{ Conflicts rate for systems $n=70$, Run time $=300$ seconds } \\
\hline & \multicolumn{4}{|c|}{ Before Method } & \multicolumn{4}{|c|}{ After Method } & \multirow{3}{*}{$\begin{array}{c}\# \\
\text { conflicts } \\
\text { Before } \\
\text { Method }\end{array}$} & \multirow{3}{*}{$\begin{array}{c}\# \\
\text { conflicts } \\
\text { After } \\
\text { Method }\end{array}$} \\
\hline & \multicolumn{3}{|c|}{$\begin{array}{l}\text { clusters conflicts } \\
\text { in code }\end{array}$} & \multirow{2}{*}{$\begin{array}{c}\# \\
\text { conflicts }\end{array}$} & \multicolumn{3}{|c|}{$\begin{array}{l}\text { clusters conflicts } \\
\text { in code }\end{array}$} & \multirow{2}{*}{$\begin{array}{c}\# \\
\text { conflicts }\end{array}$} & & \\
\hline & C1 & $\mathrm{C} 2$ & C3 & & C1 & C2 & C3 & & & \\
\hline 1 & 0.417 & 0.250 & 0.333 & 0.222 & 0.250 & 0.167 & 0.167 & 0.130 & 0.153 & 0.145 \\
\hline 2 & 0.333 & 0.333 & 0.333 & 0.255 & 0.083 & 0.167 & 0.167 & 0.106 & 0.152 & 0.136 \\
\hline 3 & 0.455 & 0.273 & 0.273 & 0.244 & 0.182 & 0.091 & 0.091 & 0.089 & 0.150 & 0.125 \\
\hline 4 & 0.300 & 0.300 & 0.400 & 0.263 & 0.100 & 0.100 & 0.200 & 0.105 & 0.139 & 0.122 \\
\hline 5 & 0.333 & 0.222 & 0.444 & 0.265 & 0.111 & 0.111 & 0.111 & 0.088 & 0.140 & 0.121 \\
\hline 6 & 0.250 & 0.375 & 0.375 & 0.267 & 0.125 & 0.125 & 0.125 & 0.100 & 0.137 & 0.127 \\
\hline 7 & 0.375 & 0.250 & 0.375 & 0.320 & 0.125 & 0.000 & 0.125 & 0.080 & 0.126 & 0.116 \\
\hline 8 & 0.167 & 0.667 & 0.167 & 0.316 & 0.000 & 0.167 & 0.000 & 0.053 & 0.148 & 0.125 \\
\hline 9 & 0.400 & 0.600 & 0.000 & 0.294 & 0.000 & 0.200 & 0.000 & 0.059 & 0.146 & 0.110 \\
\hline 10 & 0.000 & 0.400 & 0.600 & 0.333 & 0.000 & 0.000 & 0.200 & 0.067 & 0.157 & 0.114 \\
\hline
\end{tabular}




\section{Table 5 (on next page)}

Glest Conflicts rate in security generated before and after implementing the SSBFCSOS vs. OBFSoS algorithms 


\begin{tabular}{|c|c|c|c|c|c|c|c|c|c|c|}
\hline \multirow{5}{*}{$\begin{array}{c}\text { Glest } \\
\text { Simulation } \\
\text { Attempts }\end{array}$} & \multicolumn{8}{|c|}{ SSBFCSoS } & \multicolumn{2}{|c|}{ OBFSoS } \\
\hline & \multicolumn{10}{|c|}{ Conflicts rate for systems $n=70$, Run time $=300$ seconds } \\
\hline & \multicolumn{4}{|c|}{ Before Method } & \multicolumn{4}{|c|}{ After Method } & \multirow{3}{*}{$\begin{array}{c}\# \\
\text { conflicts } \\
\text { Before } \\
\text { Method }\end{array}$} & \multirow{3}{*}{$\begin{array}{c}\# \\
\text { conflicts } \\
\text { After } \\
\text { Method }\end{array}$} \\
\hline & \multicolumn{3}{|c|}{$\begin{array}{l}\text { clusters conflicts } \\
\text { in security }\end{array}$} & \multirow{2}{*}{$\begin{array}{c}\# \\
\text { conflicts }\end{array}$} & \multicolumn{3}{|c|}{$\begin{array}{l}\text { clusters conflicts } \\
\text { in security }\end{array}$} & \multirow{2}{*}{$\begin{array}{c}\# \\
\text { conflicts }\end{array}$} & & \\
\hline & C1 & C2 & C3 & & C1 & C2 & C3 & & & \\
\hline 1 & 0.444 & 0.222 & 0.333 & 0.167 & 0.333 & 0.111 & 0.222 & 0.111 & 0.168 & 0.153 \\
\hline 2 & 0.375 & 0.375 & 0.250 & 0.170 & 0.250 & 0.250 & 0.125 & 0.106 & 0.160 & 0.152 \\
\hline 3 & 0.286 & 0.286 & 0.429 & 0.156 & 0.143 & 0.143 & 0.286 & 0.089 & 0.158 & 0.142 \\
\hline 4 & 0.500 & 0.500 & 0.000 & 0.158 & 0.333 & 0.333 & 0.000 & 0.105 & 0.165 & 0.130 \\
\hline 5 & 0.400 & 0.200 & 0.400 & 0.147 & 0.200 & 0.200 & 0.200 & 0.088 & 0.159 & 0.131 \\
\hline 6 & 0.200 & 0.400 & 0.400 & 0.167 & 0.200 & 0.200 & 0.000 & 0.067 & 0.157 & 0.127 \\
\hline 7 & 0.750 & 0.250 & 0.000 & 0.160 & 0.250 & 0.250 & 0.000 & 0.080 & 0.147 & 0.126 \\
\hline 8 & 0.000 & 0.667 & 0.333 & 0.158 & 0.000 & 0.333 & 0.000 & 0.053 & 0.159 & 0.125 \\
\hline 9 & 0.500 & 0.250 & 0.250 & 0.235 & 0.250 & 0.000 & 0.000 & 0.059 & 0.146 & 0.122 \\
\hline 10 & 0.667 & 0.000 & 0.333 & 0.200 & 0.000 & 0.000 & 0.000 & 0.067 & 0.143 & 0.114 \\
\hline
\end{tabular}




\section{Table 6(on next page)}

Glest \# of resolved conflicts rate generated SSBFCSoS vs. OBFSoS with consumed time 


\begin{tabular}{c|c|c|c|c}
\hline \multirow{2}{*}{$\begin{array}{c}\text { Glest } \\
\text { simulation } \\
\text { attempts }\end{array}$} & Rate & Time & Rate & Time \\
\cline { 2 - 5 } $\mathbf{1}$ & 0.296 & 281.11 & 0.076 & 300.00 \\
\hline $\mathbf{2}$ & 0.319 & 270.59 & 0.088 & 300.00 \\
\hline $\mathbf{3}$ & 0.422 & 262.28 & 0.133 & 288.89 \\
\hline $\mathbf{4}$ & 0.421 & 214.67 & 0.165 & 278.26 \\
\hline $\mathbf{5}$ & 0.471 & 204.44 & 0.15 & 283.49 \\
\hline $\mathbf{6}$ & 0.533 & 180.39 & 0.167 & 277.78 \\
\hline $\mathbf{7}$ & 0.560 & 161.40 & 0.189 & 270.18 \\
\hline $\mathbf{8}$ & 0.684 & 161.85 & 0.159 & 280.30 \\
\hline $\mathbf{9}$ & 0.706 & 122.34 & 0.183 & 272.36 \\
\hline $\mathbf{1 0}$ & 0.733 & 113.58 & 0.171 & 276.19 \\
\hline
\end{tabular}




\section{Table 7 (on next page)}

StarCraft Brood War Conflicts rate generated for SSBFCSoS vs. OBFSoS 


\begin{tabular}{|c|c|c|c|c||c|c|c|c|}
\hline \multirow{2}{*}{$\begin{array}{c}\text { StarCraft } \\
\text { Brood } \\
\text { War } \\
\text { simulation } \\
\text { attempts }\end{array}$} & \multicolumn{5}{|c||}{ SSBFCSoS } & \multicolumn{4}{c|}{ OBFSoS } \\
\cline { 2 - 9 } & \multicolumn{4}{|c||}{ Conflicts rate for systems n = 100, Run time = 400 seconds } \\
\cline { 2 - 9 } & decision & design & security & code & decision & design & security & code \\
\hline $\mathbf{1}$ & 0.343 & 0.239 & 0.209 & 0.209 & 0.364 & 0.283 & 0.192 & 0.162 \\
\hline $\mathbf{2}$ & 0.322 & 0.254 & 0.203 & 0.220 & 0.385 & 0.275 & 0.181 & 0.159 \\
\hline $\mathbf{3}$ & 0.314 & 0.255 & 0.216 & 0.216 & 0.390 & 0.273 & 0.174 & 0.163 \\
\hline $\mathbf{4}$ & 0.319 & 0.255 & 0.191 & 0.234 & 0.383 & 0.272 & 0.185 & 0.160 \\
\hline $\mathbf{5}$ & 0.317 & 0.268 & 0.195 & 0.220 & 0.396 & 0.268 & 0.181 & 0.154 \\
\hline $\mathbf{6}$ & 0.303 & 0.242 & 0.212 & 0.242 & 0.396 & 0.273 & 0.180 & 0.151 \\
\hline $\mathbf{7}$ & 0.333 & 0.222 & 0.222 & 0.222 & 0.398 & 0.273 & 0.172 & 0.156 \\
\hline $\mathbf{8}$ & 0.333 & 0.250 & 0.167 & 0.250 & 0.435 & 0.287 & 0.204 & 0.074 \\
\hline $\mathbf{9}$ & 0.250 & 0.300 & 0.200 & 0.250 & 0.394 & 0.279 & 0.173 & 0.154 \\
\hline $\mathbf{1 0}$ & 0.278 & 0.278 & 0.222 & 0.222 & 0.402 & 0.278 & 0.186 & 0.134 \\
\hline
\end{tabular}




\section{Table 8 (on next page)}

StarCraft Brood War Resolved Conflicts generated after implementing the SSBFCSoS vs. OBFSOS 


\begin{tabular}{|c|c|c|c|c||c|c|c|c|}
\hline \multirow{2}{*}{$\begin{array}{c}\text { StarCraft } \\
\text { Brood } \\
\text { War } \\
\text { simulation } \\
\text { attempts }\end{array}$} & \multicolumn{4}{|c||}{ SSBFCSoS } & \multicolumn{4}{c|}{ OBFSoS } \\
\cline { 2 - 9 } & \multicolumn{4}{|c||}{ Conflicts rate for systems n = 100, Run time = 400 seconds } \\
\cline { 2 - 9 } & decision & design & security & code & decision & design & security & code \\
\hline $\mathbf{1}$ & 0.269 & 0.224 & 0.164 & 0.149 & 0.343 & 0.258 & 0.177 & 0.157 \\
\hline $\mathbf{2}$ & 0.254 & 0.220 & 0.169 & 0.153 & 0.352 & 0.264 & 0.181 & 0.159 \\
\hline $\mathbf{3}$ & 0.255 & 0.235 & 0.157 & 0.157 & 0.355 & 0.250 & 0.163 & 0.163 \\
\hline $\mathbf{4}$ & 0.255 & 0.234 & 0.149 & 0.149 & 0.352 & 0.241 & 0.167 & 0.167 \\
\hline $\mathbf{5}$ & 0.268 & 0.195 & 0.146 & 0.122 & 0.362 & 0.248 & 0.161 & 0.161 \\
\hline $\mathbf{6}$ & 0.212 & 0.182 & 0.152 & 0.121 & 0.367 & 0.245 & 0.158 & 0.158 \\
\hline $\mathbf{7}$ & 0.222 & 0.185 & 0.148 & 0.111 & 0.375 & 0.242 & 0.156 & 0.156 \\
\hline $\mathbf{8}$ & 0.208 & 0.208 & 0.125 & 0.083 & 0.389 & 0.259 & 0.176 & 0.167 \\
\hline $\mathbf{9}$ & 0.100 & 0.200 & 0.100 & 0.100 & 0.365 & 0.240 & 0.154 & 0.163 \\
\hline $\mathbf{1 0}$ & 0.056 & 0.056 & 0.111 & 0.111 & 0.309 & 0.227 & 0.155 & 0.144 \\
\hline
\end{tabular}




\section{Table 9 (on next page)}

StarCraft Brood War Resolved Conflicts rate generated after implementing the SSBFCSOS vs. OBFSOS with consumed time 


\begin{tabular}{c|c|c|c|c}
\hline \multirow{2}{*}{$\begin{array}{c}\text { StarCraft Brood } \\
\text { War } \\
\text { simulation } \\
\text { attempts }\end{array}$} & Rate & Time & Rate & Time \\
\cline { 2 - 5 } & 0.194 & 369.15 & 0.066 & 399.28 \\
\hline $\mathbf{1}$ & 0.203 & 251.26 & 0.044 & 377.43 \\
\hline $\mathbf{2}$ & 0.196 & 198.5 & 0.070 & 370.44 \\
\hline $\mathbf{3}$ & 0.213 & 189.34 & 0.074 & 355.31 \\
\hline $\mathbf{5}$ & 0.268 & 158.55 & 0.067 & 341.37 \\
\hline $\mathbf{6}$ & 0.333 & 131.51 & 0.072 & 335.58 \\
\hline $\mathbf{7}$ & 0.333 & 127.28 & 0.070 & 317.08 \\
\hline $\mathbf{8}$ & 0.375 & 101.45 & 0.009 & 304.21 \\
\hline $\mathbf{9}$ & 0.500 & 98.56 & 0.077 & 299.25 \\
\hline $\mathbf{1 0}$ & 0.667 & 87.54 & 0.165 & 289.11 \\
\hline
\end{tabular}




\section{Table $\mathbf{1 0}$ (on next page)}

StarCraft Brood War Resolving Conflicts rate generated after implementing the SSBFCSoS method using k-means clustering vs. Agglomerative (bottom-up) with consumed time 


\begin{tabular}{c|c|c|c|c}
\hline \multirow{2}{*}{\begin{tabular}{c} 
\# systems \\
\cline { 2 - 5 }
\end{tabular}} & \multicolumn{2}{|c|}{ k-means } & \multicolumn{2}{c}{ Agglomerative (bottom-up) } \\
\cline { 2 - 5 } & Rate & Time & Rate & Time \\
\hline $\mathbf{2 0}$ & 0.846 & 60.42 & 0.692 & 99.52 \\
\hline $\mathbf{3 5}$ & 0.778 & 99.38 & 0.704 & 161.55 \\
\hline $\mathbf{6 5}$ & 0.895 & 121.05 & 0.667 & 232.32 \\
\hline $\mathbf{8 0}$ & 0.855 & 196.57 & 0.696 & 333.33 \\
\hline $\mathbf{9 5}$ & 0.884 & 241.47 & 0.663 & 423.45 \\
\hline $\mathbf{1 1 0}$ & 0.899 & 279.42 & 0.687 & 789.50 \\
\hline $\mathbf{1 3 0}$ & 0.888 & 329.36 & 0.664 & 953.36 \\
\hline $\mathbf{1 4 0}$ & 0.876 & 373.16 & 0.664 & 1696.17 \\
\hline $\mathbf{1 7 0}$ & 0.884 & 406.38 & 0.661 & 1900.53 \\
\hline $\mathbf{1 9 0}$ & 0.891 & 539.47 & 0.652 & 2884.42 \\
\hline
\end{tabular}




\section{Table 11 (on next page)}

Comparison between the k-means algorithm and some clustering algorithms 


\begin{tabular}{cccccc}
\hline Algorithm & $\begin{array}{c}\text { Dataset } \\
\text { size }\end{array}$ & Scalability & $\begin{array}{c}\text { Overlapping } \\
\text { Clusters } \\
\text { (Conflicts) }\end{array}$ & $\begin{array}{c}\text { Time } \\
\text { Consuming }\end{array}$ & $\begin{array}{c}\text { Adapting } \\
\text { with SoS/IoT }\end{array}$ \\
\hline k-means & Huge & Yes & No & Low & Yes \\
K-medoids & Small & No & Yes & High & No \\
CLARA & Huge & Yes & No & Medium & Yes \\
Agglomerative & Huge & Yes & No & High & Yes \\
(bottom-up) & Huge & No & - & Medium & Yes \\
STING & Medium & Yes & Yes & High & No \\
c-means & Huge & Yes & - & High & No \\
BIRCH & Huge & Yes & - & High & Yes \\
CURE & Huge & Yes & No & High & No \\
CHAMELEON & Small & No & No & Medium & Yes \\
OPTICS & Huge & Yes & No & High & No \\
HASTREAM & Small & No & No & High & No \\
Liar Tree & Small & No & Yes & High & Yes \\
\hline SOM & & & & & \\
\hline
\end{tabular}

1

2

3 
Figure 1

The proposed Method 


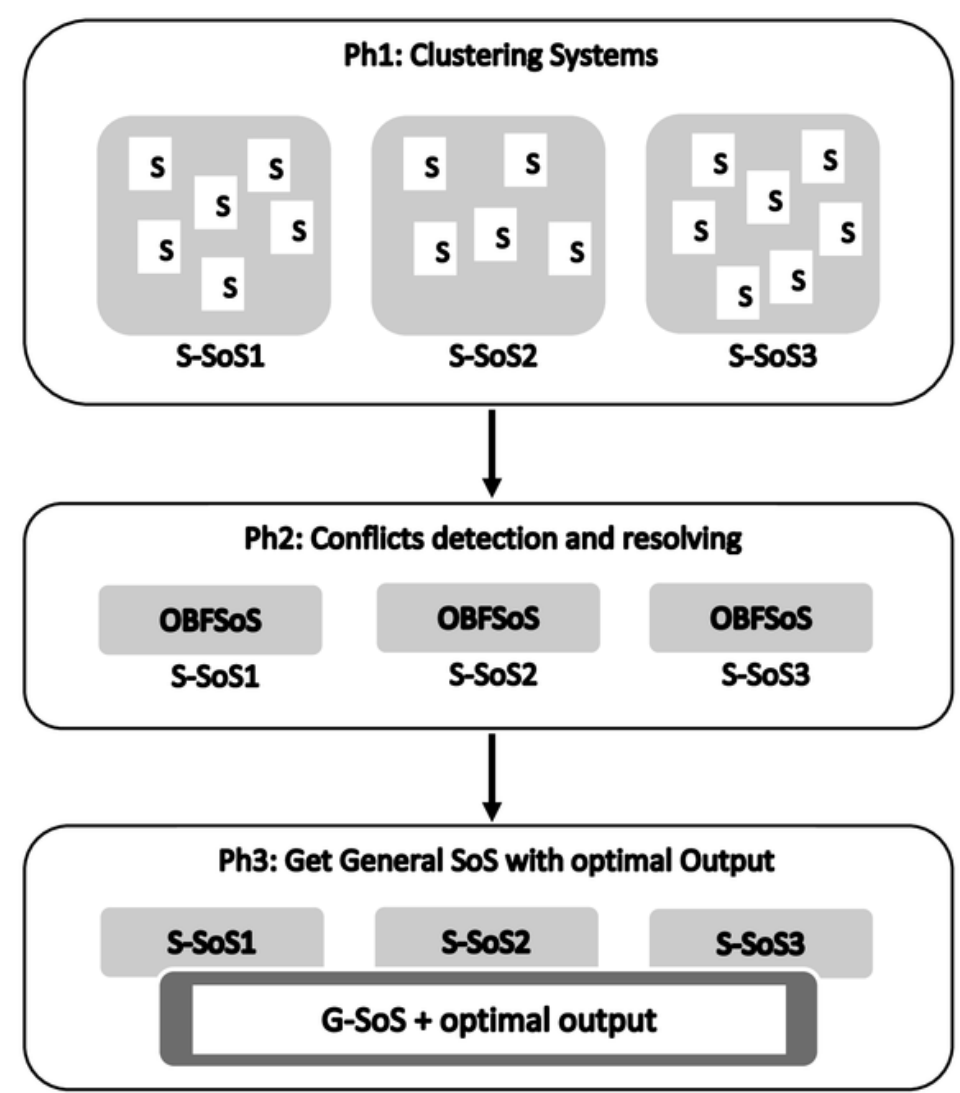


Figure 2

Conflicts rate in Glest for SSBFCSoS vs. OBFSoS 


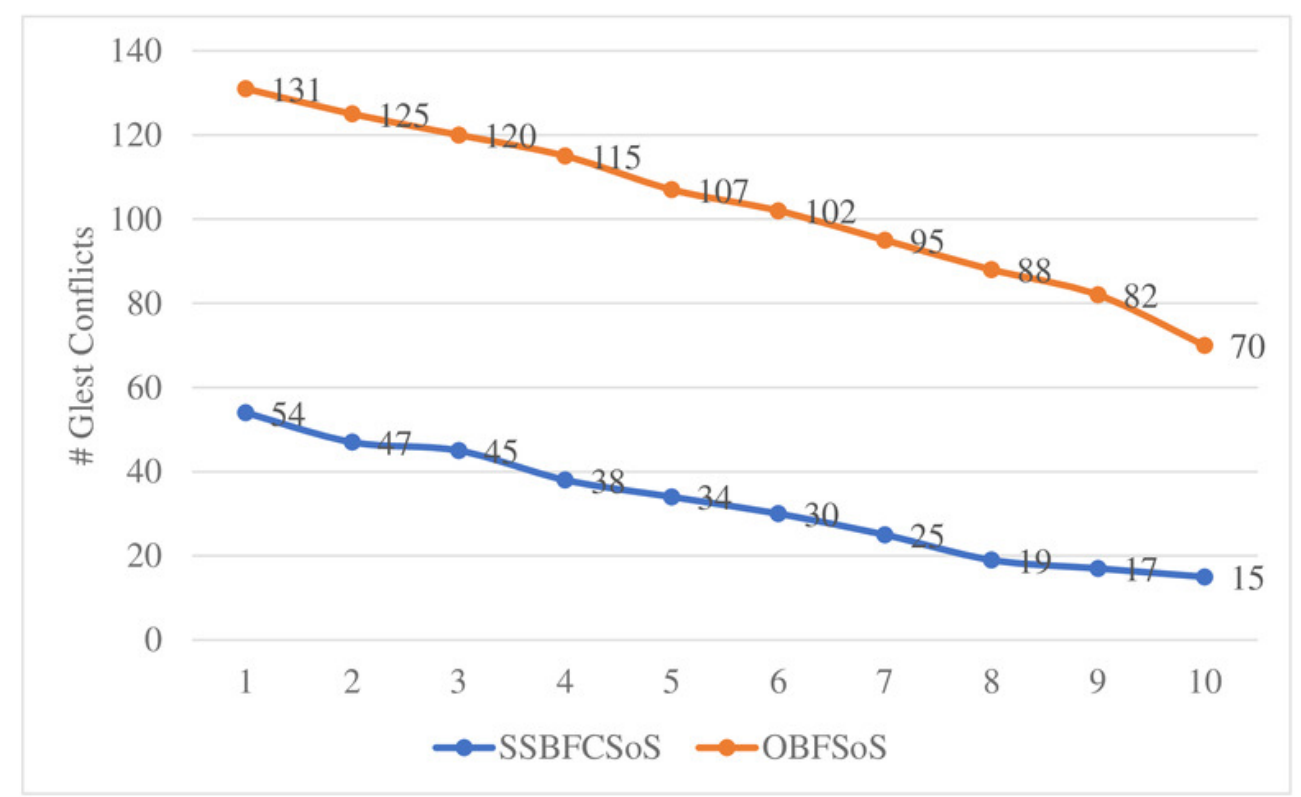


Figure 3

Glest Conflicts rate in decisions after implementing SSBFCSoS vs. OBFSoS 


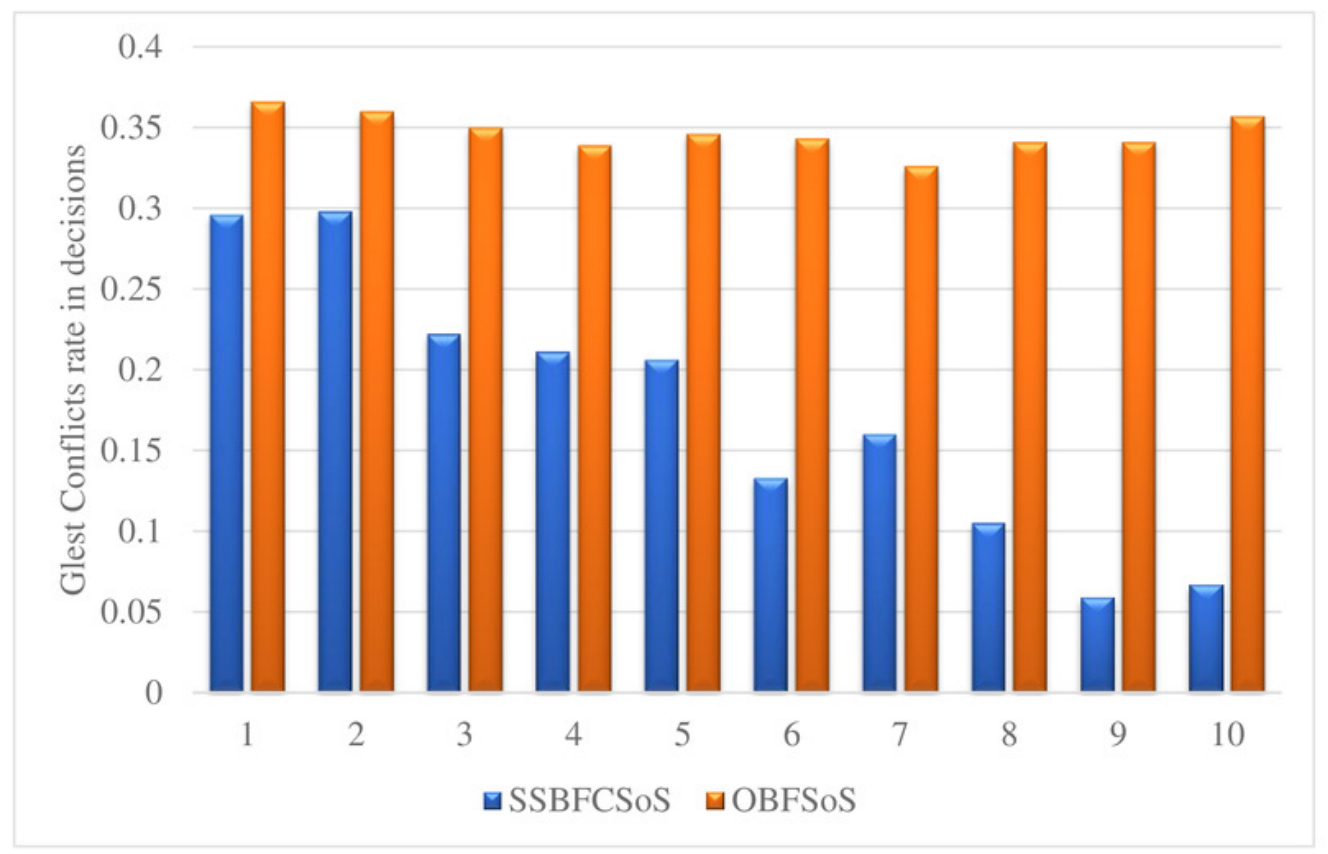


Figure 4

Glest Conflicts rate in design after implementing SSBFCSoS vs. OBFSoS 


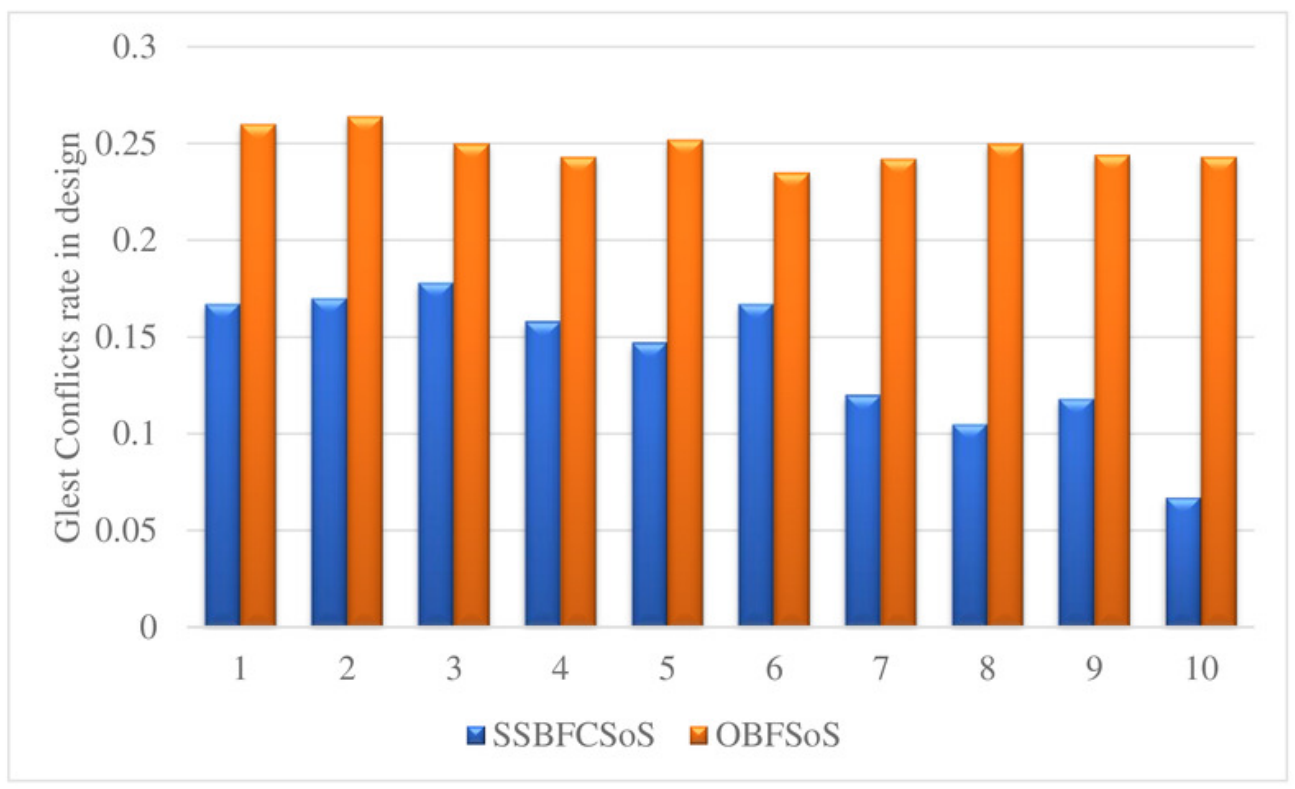


Figure 5

Glest Conflicts rate in code after implementing SSBFCSoS vs. OBFSoS 


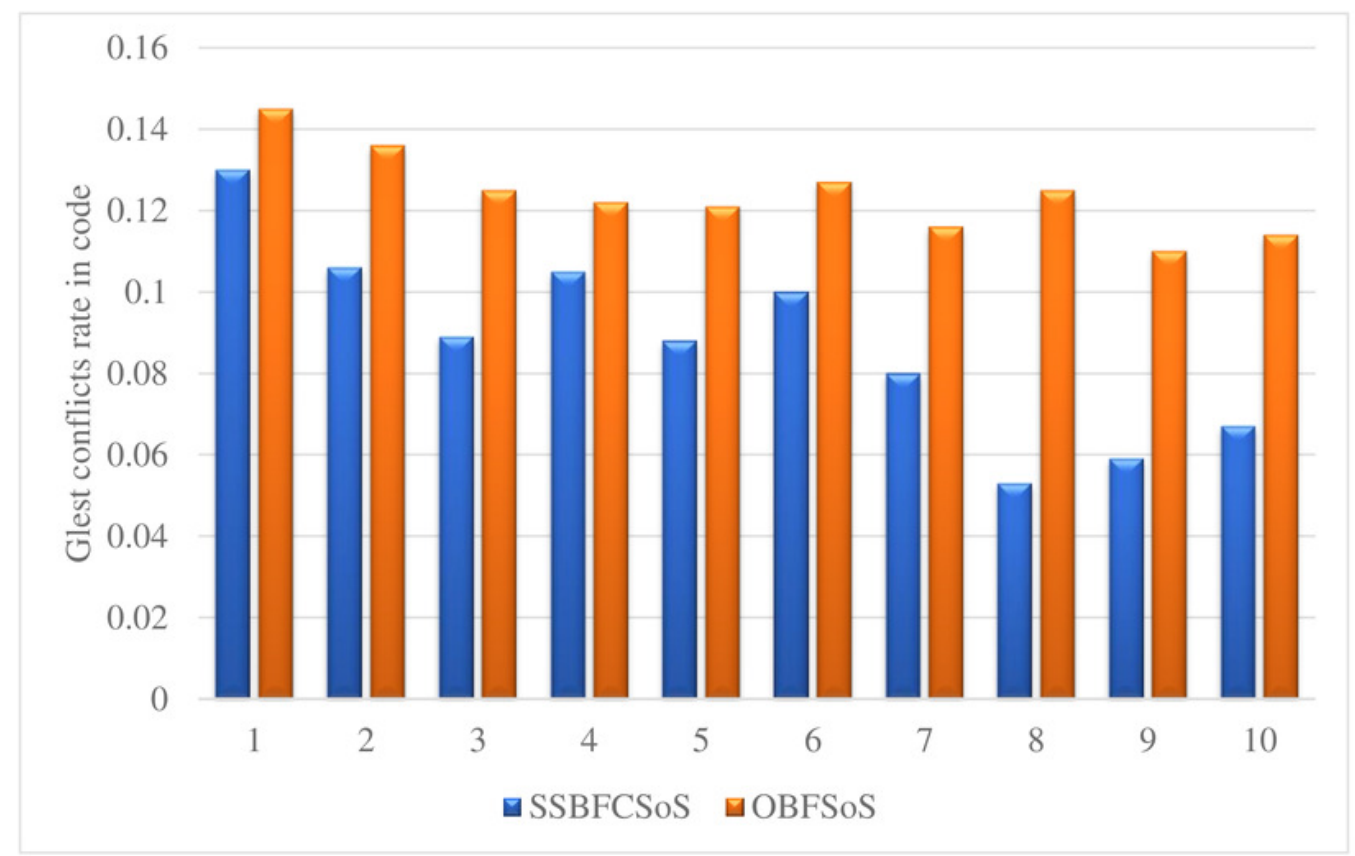


Figure 6

Glest Conflicts rate in security after implementing SSBFCSOS vs. OBFSoS 


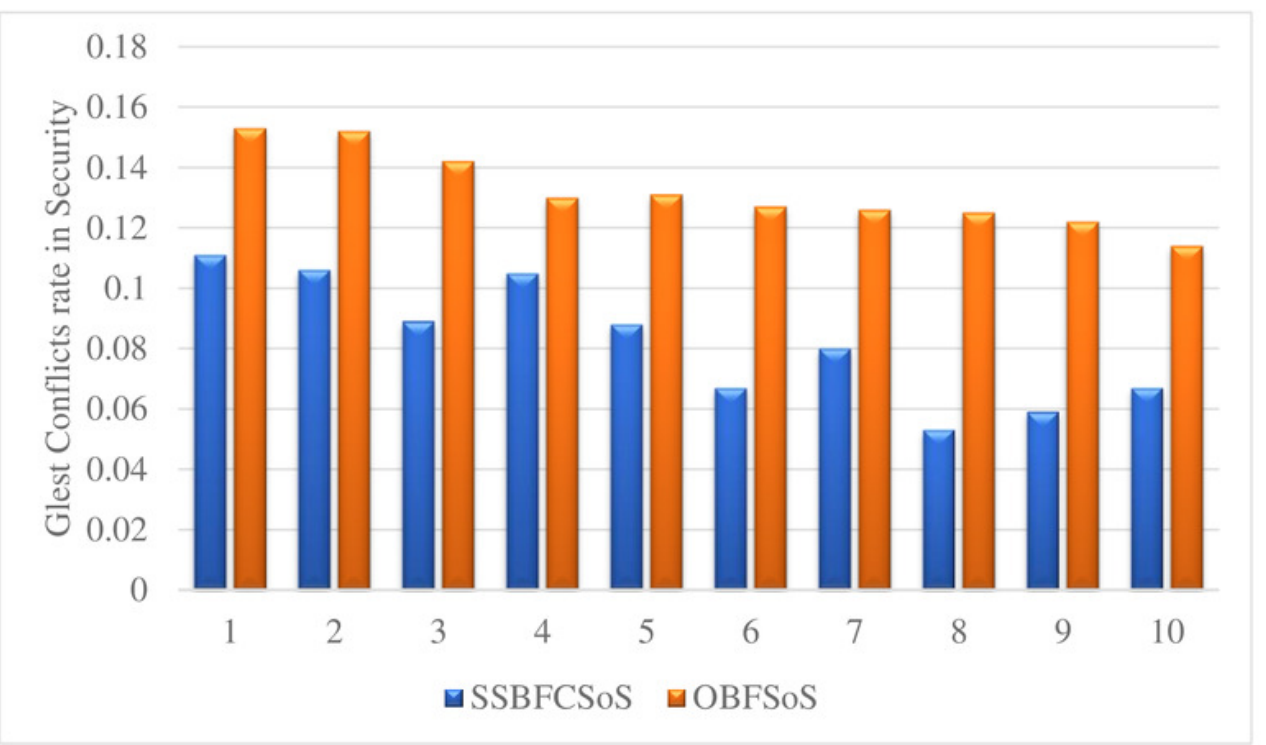




\section{Figure 7}

Glest Resolved Conflicts rate after implementing SSBFCSoS vs. OBFSoS 


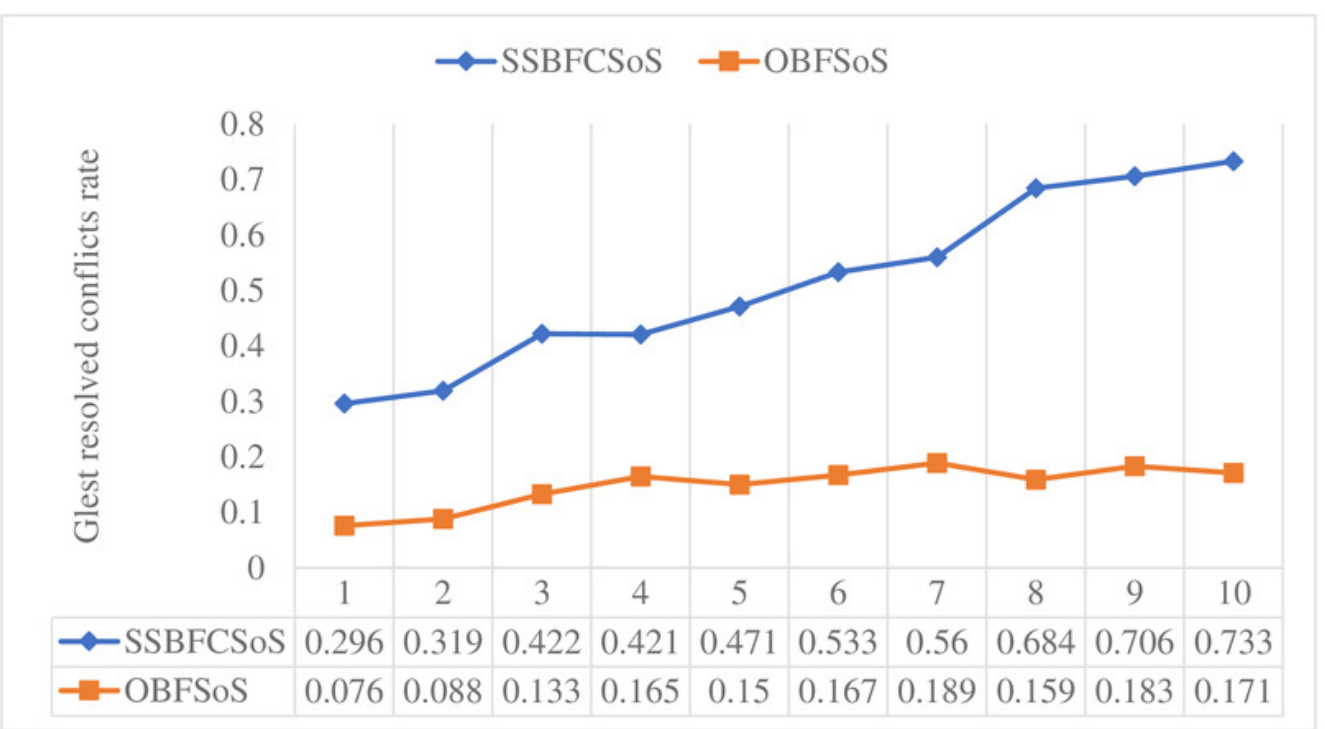


Figure 8

Glest Time Consumed in Conflicts Resolving during implementing SSBFCSOS vs. OBFSoS 


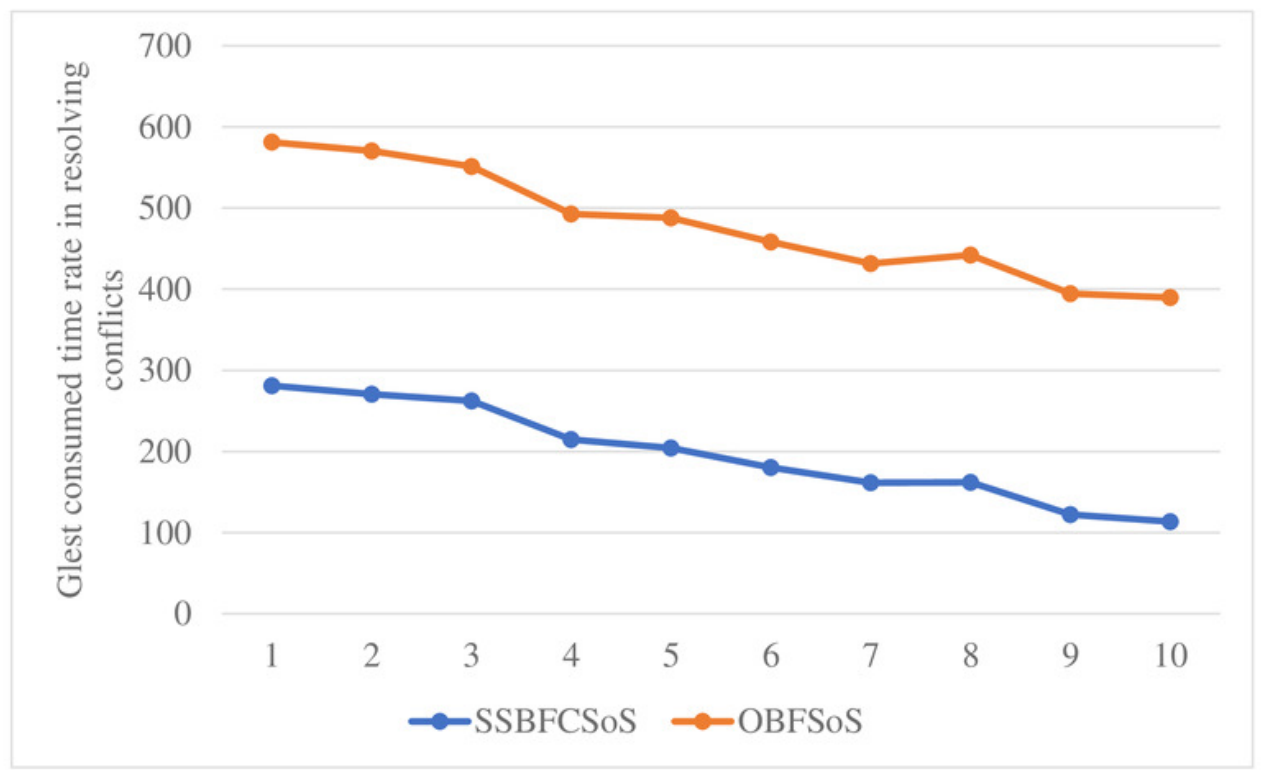


Figure 9

StarCraft Brood War Conflicts rate in decisions after implementing SSBFCSoS vs. OBFSOS 


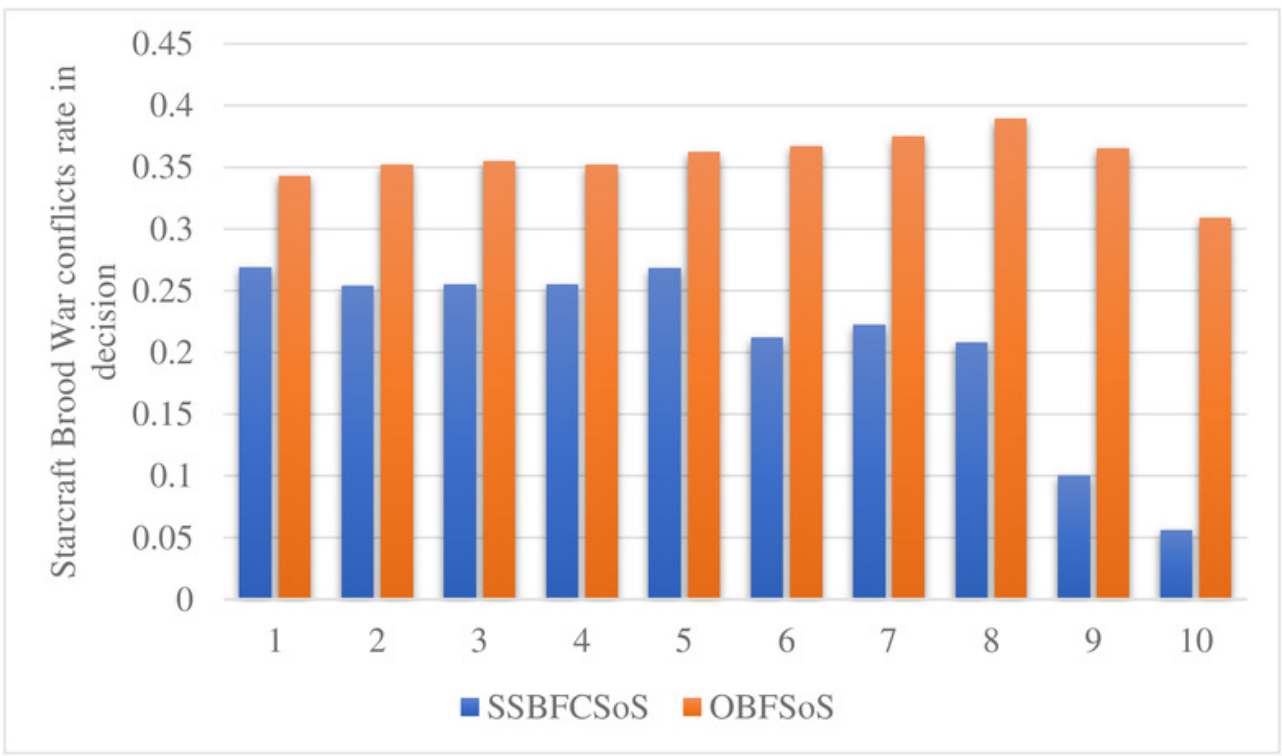


Figure 10

StarCraft Brood War Conflicts rate in design after implementing SSBFCSoS vs. OBFSoS 


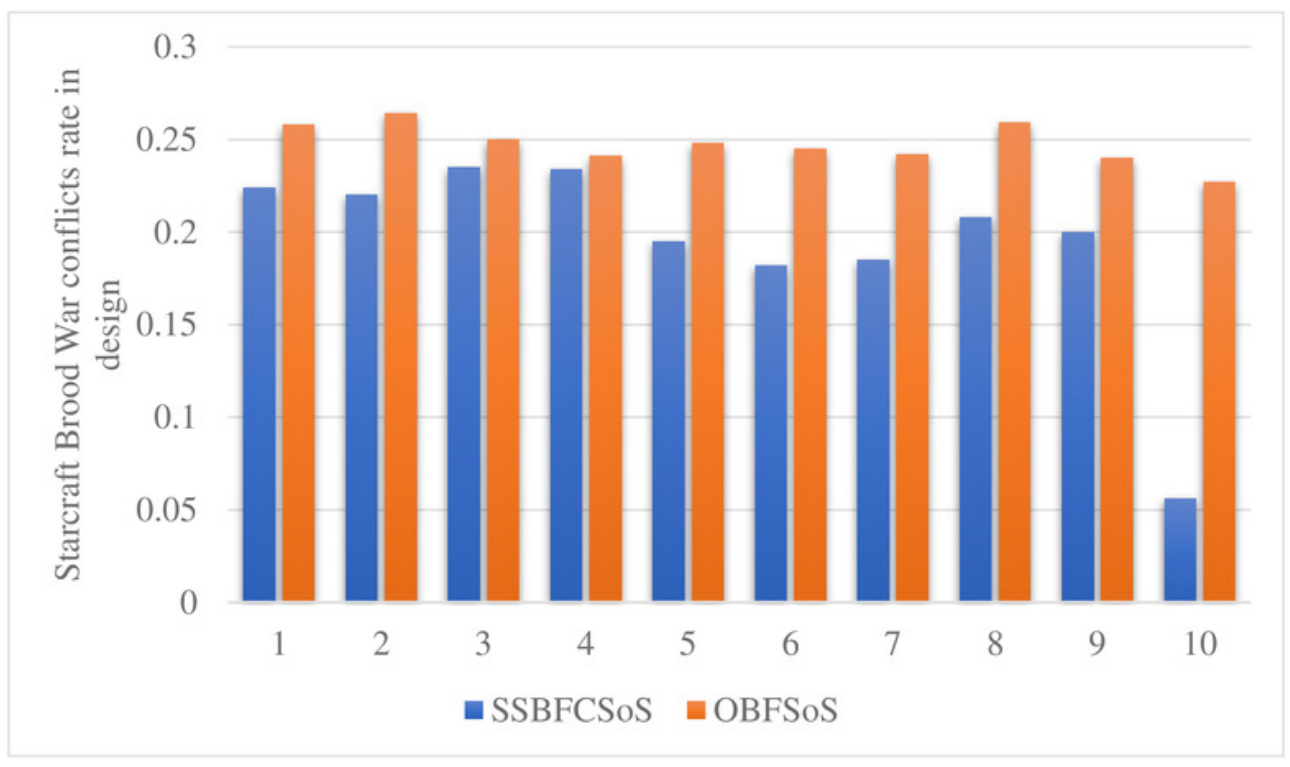


Figure 11

StarCraft Brood War Conflicts rate in security after implementing SSBFCSoS vs. OBFSoS 


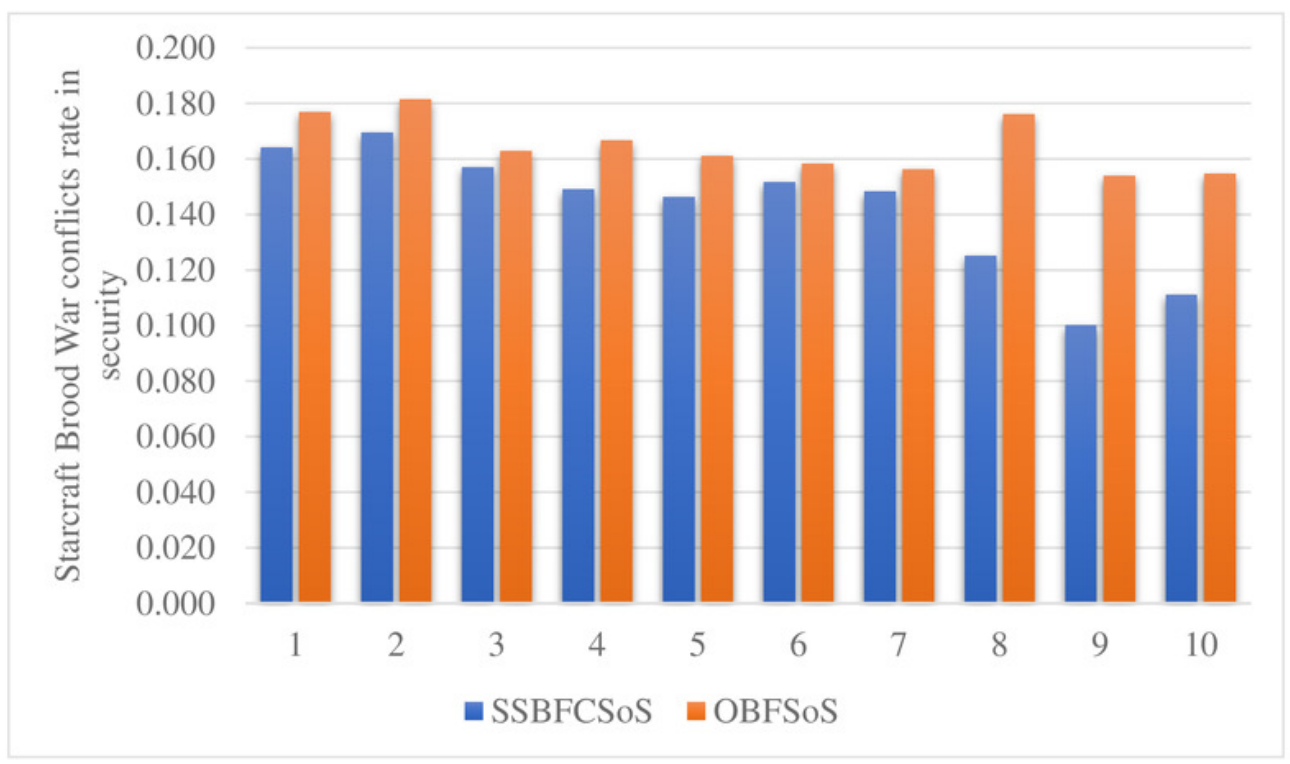




\section{Figure 12}

StarCraft Brood War Conflicts rate in code after implementing SSBFCSOS vs. OBFSoS 


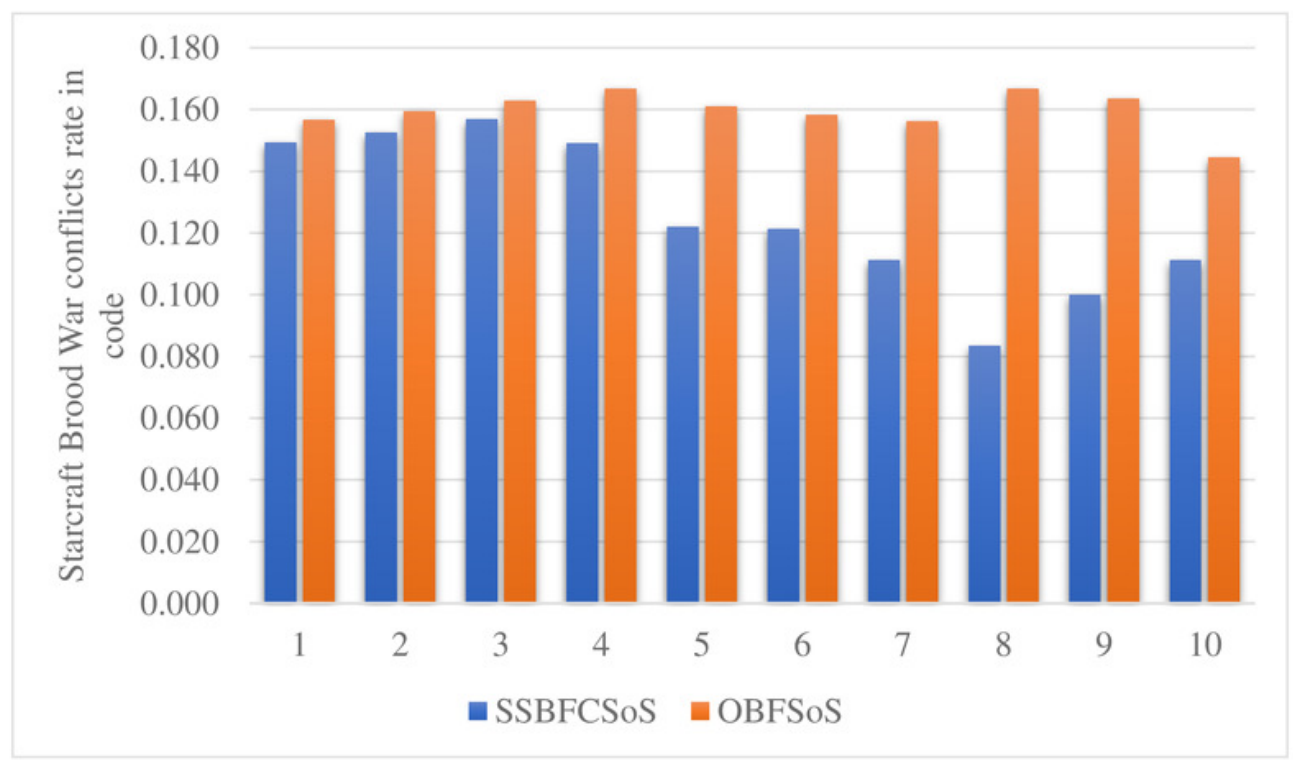


Figure 13

StarCraft Brood War Resolved Conflicts rate after implementing SSBFCSoS vs. OBFSoS 


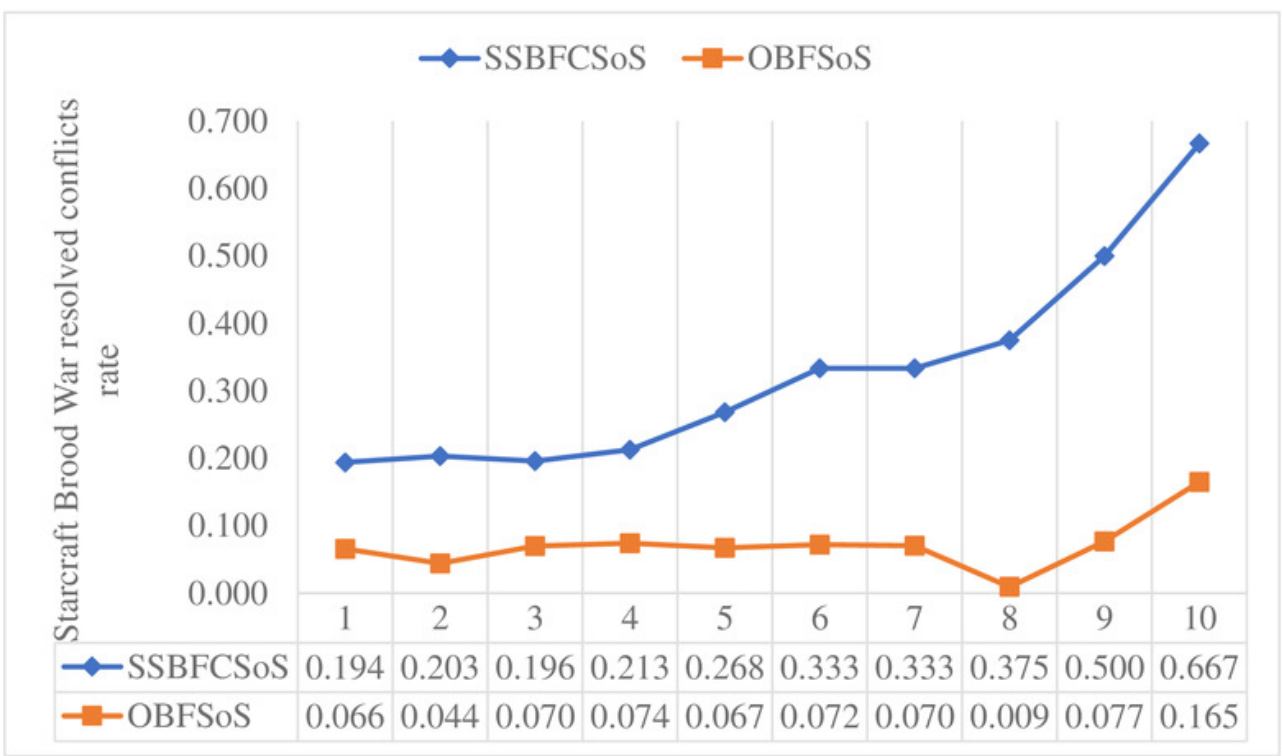


Figure 14

StarCraft Brood War Time Consumed in Conflicts Resolving during implementing SSBFCSOS vs. OBFSOS 


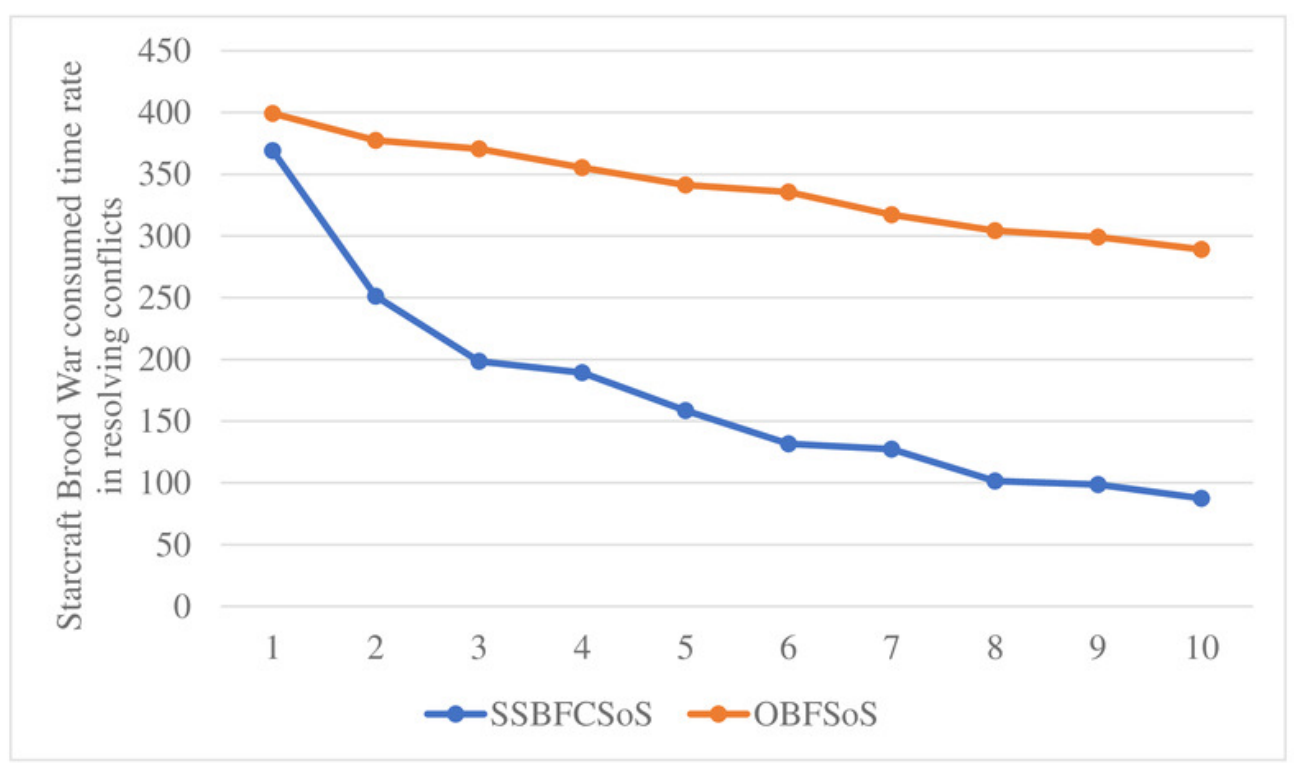


Figure 15

Resolved Conflicts rate for SSBFCSoS with different cluster numbers and systems 


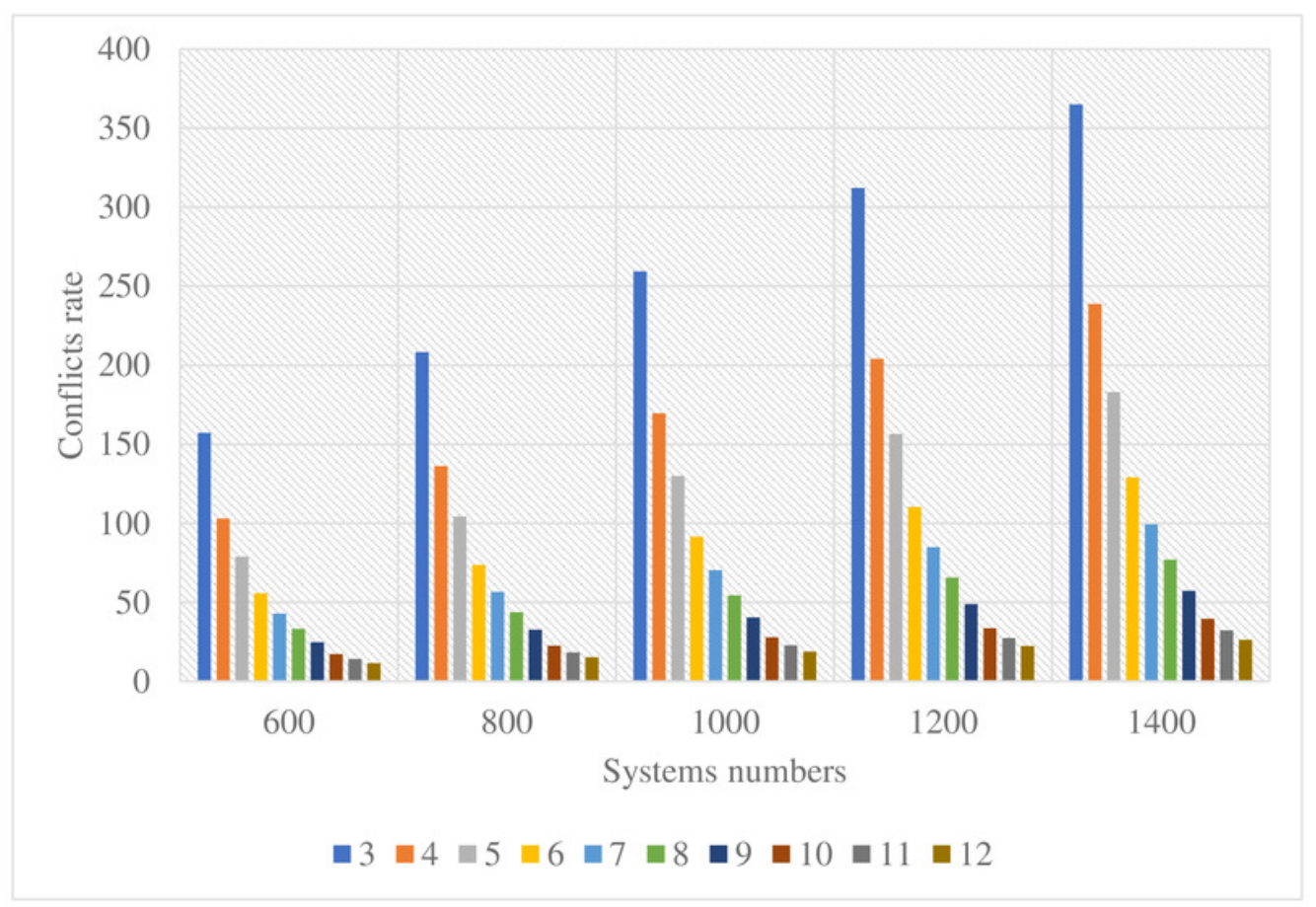




\section{Figure 16}

StarCraft Brood War Resolved Conflicts rate generated implementing the SSBFCSoS using k-means clustering vs. Agglomerative (bottom-up) 


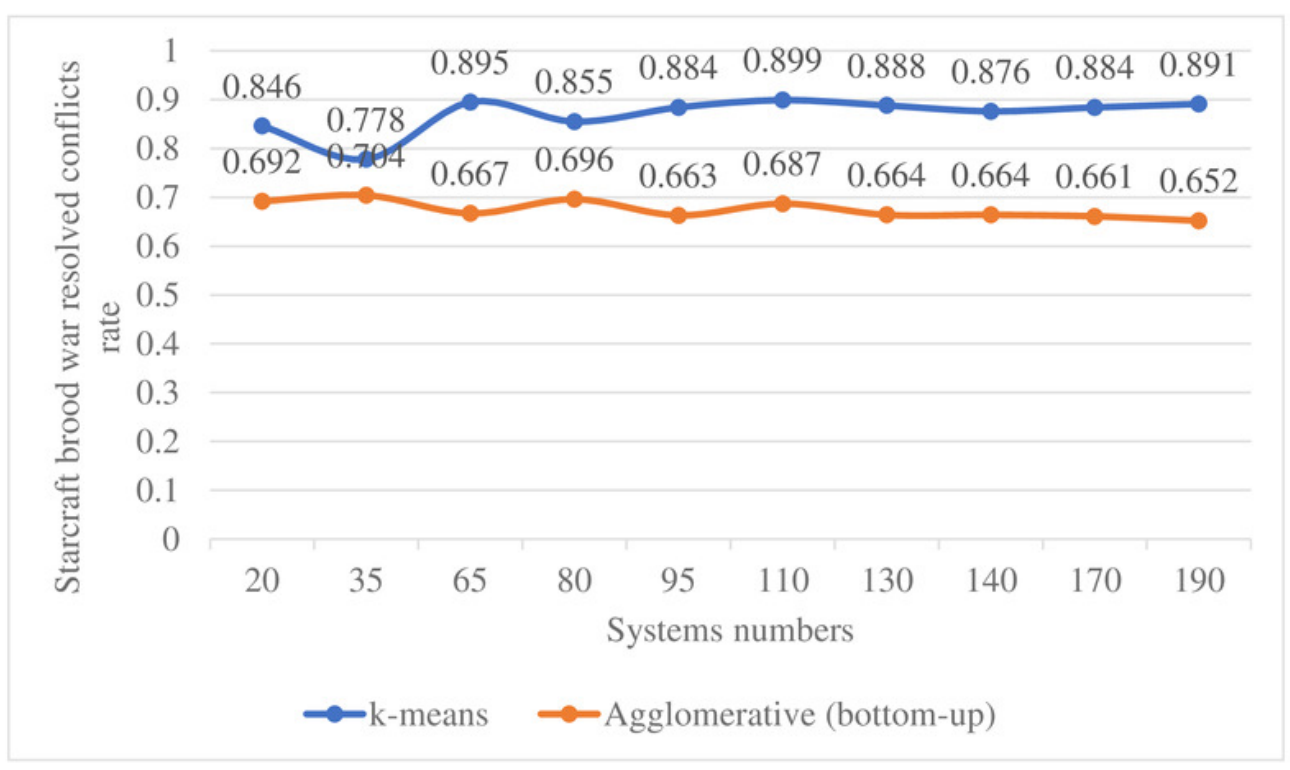


Figure 17

StarCraft Brood War Time Consumed in Conflicts Resolving during implementing SSBFCSoS using k-means vs. Agglomerative (bottom-up) clustering techniques 


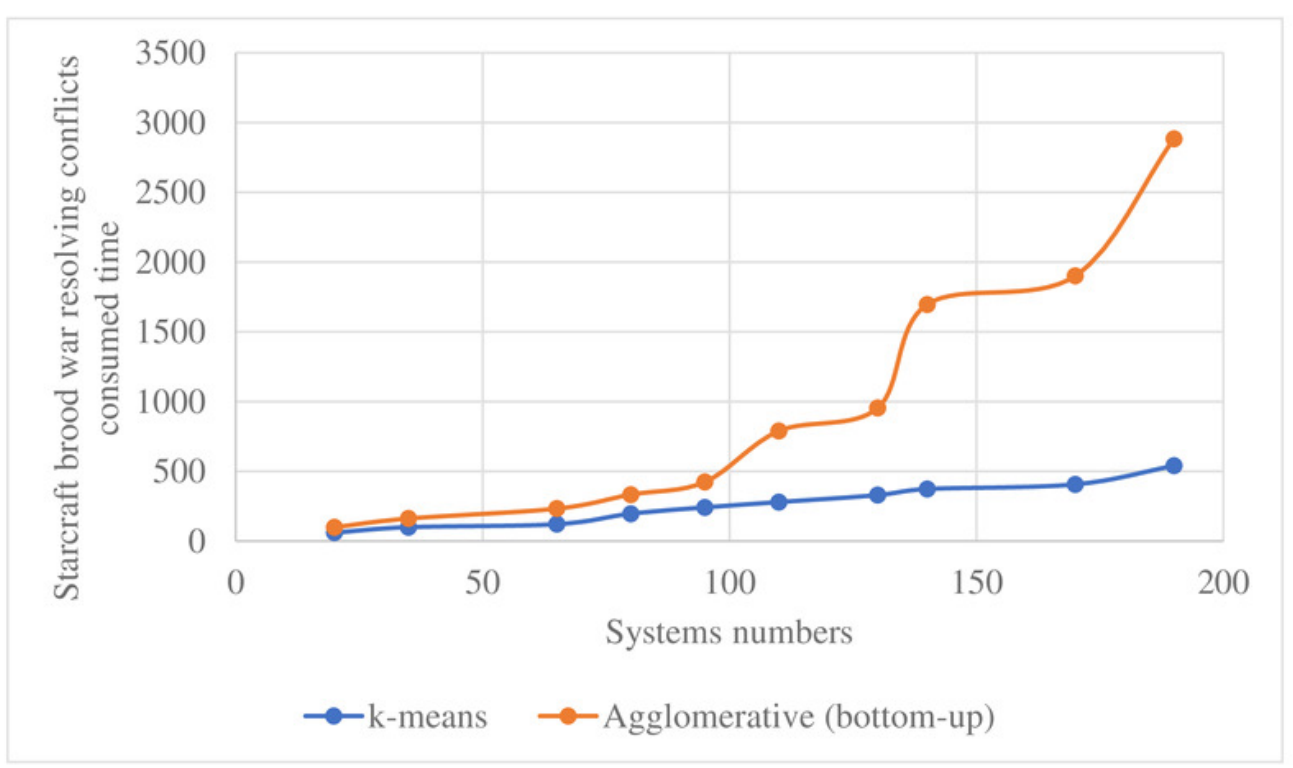

